\title{
Bridge number and integral Dehn surgery
}

\author{
KENNETH L BAKER \\ CAMERON GORDON \\ JOHN LUECKE
}

In a 3-manifold $M$, let $K$ be a knot and $\hat{R}$ be an annulus which meets $K$ transversely. We define the notion of the pair $(\hat{R}, K)$ being caught by a surface $Q$ in the exterior of the link $K \cup \partial \widehat{R}$. For a caught pair $(\widehat{R}, K)$, we consider the knot $K^{n}$ gotten by twisting $K n$ times along $\hat{R}$ and give a lower bound on the bridge number of $K^{n}$ with respect to Heegaard splittings of $M$; as a function of $n$, the genus of the splitting, and the catching surface $Q$. As a result, the bridge number of $K^{n}$ tends to infinity with $n$. In application, we look at a family of knots $\left\{K^{n}\right\}$ found by Teragaito that live in a small Seifert fiber space $M$ and where each $K^{n}$ admits a Dehn surgery giving $S^{3}$. We show that the bridge number of $K^{n}$ with respect to any genus-2 Heegaard splitting of $M$ tends to infinity with $n$. This contrasts with other work of the authors as well as with the conjectured picture for knots in lens spaces that admit Dehn surgeries giving $S^{3}$.

$57 \mathrm{M} 25,57 \mathrm{M} 27$

\section{Introduction}

One may produce a family $\left\{K^{n}\right\}$ of knots in an orientable 3-manifold $M$ by Dehn twisting a knot $K=K^{0}$ along an annulus $\widehat{R}$ that it intersects transversely. If $\widehat{R}$ lies in a genus- $g$ Heegaard surface for $M$ then $\mathfrak{b}_{g}\left(K^{n}\right)$, the minimal bridge number of $K^{n}$ among genus- $g$ Heegaard splittings of $M$, is bounded. We give sufficient conditions to guarantee a converse to this statement. This allows us to give examples of knots in the $3-$ sphere whose integral Dehn surgeries contrast with our results in $[2 ; 1]$ on non-integral Dehn surgeries. Specifically, when performing a Dehn surgery on a knot $K^{\prime}$ in $S^{3}$, the core of the attached solid torus becomes a knot $K$ in the resulting manifold $M$. We refer to $K^{\prime} \subset S^{3}$ and $K \subset M$ as surgery-duals. We show that there is a family of hyperbolic knots in the 3-sphere for which an integral surgery is the same genus-2 manifold $M$ and whose surgery-duals have unbounded bridge numbers with respect to any genus- 2 Heegaard splitting of $M$. 
The converse mentioned above, that an upper bound on $\mathfrak{b}_{g}\left(K^{n}\right)$ implies that the annulus $\hat{R}$ lies in a genus- $g$ Heegaard surface for $M$, requires us to find a lower bound on the bridge number of $K_{n}$ that otherwise increases with $n$. In general, it is difficult to obtain good lower bounds on bridge number. The engine of this paper is Theorem 1.3 (and in particular its supporting Lemma 2.8) which gives such a lower bound when the knot comes from twisting along an annulus.

As noted above, if $\widehat{R}$ lies in a genus- $g$ Heegaard surface for $M$ then $\mathfrak{b}_{g}\left(K^{n}\right)$ is bounded. It is easy to see that the same conclusion holds if $\partial \hat{R}$ bounds an annulus in the exterior of $K$ with the same framing at $\partial \hat{R}$ as $\hat{R}$. The following shows that if $(\hat{R}, K)$ is caught (defined below) then these are the only conditions under which $b_{g}\left(K^{n}\right)$ is bounded.

Corollary 1.1 Let $M$ be a compact, orientable 3-manifold with (possibly empty) boundary. Let $\hat{R}$ be an annulus and $K$ be a knot in $M$ such that the pair $(\hat{R}, K)$ in $M$ is caught. Fixing an orientation on $M$, let $K^{n}$ be $K$ twisted $n$ times along $\hat{R}$. Assume $M$ has a genus- $g$ Heegaard splitting. Then one of the following holds:

(1) There is a genus- $g$ Heegaard surface of $M$ containing $\widehat{R}$.

(2) There is another annulus $\hat{A}$ in $M$ that is disjoint from $K$ with $\partial \hat{A}=\partial \hat{R}$, such that $\hat{A}$ and $\hat{R}$ induce the same framing on $\partial \widehat{R}$.

(3) $\mathfrak{b}_{g}\left(K^{n}\right) \rightarrow \infty$ as $n \rightarrow \infty$.

Furthermore, if either (1) or (2) hold, then $\left\{\mathfrak{b}_{g}\left(K^{n}\right)\right\}$ is a finite set.

Definition 1.2 (Twisting along an annulus) Let $\hat{R}$ be an annulus embedded in $M$ with $\partial \hat{R}$ the link $L_{1} \cup L_{2}$ in $M$. Let $R=\widehat{R} \cap\left(M-\mathcal{N}\left(L_{1} \cup L_{2}\right)\right)$. Fix an orientation on $M$ and $\hat{R}$. This induces an orientation on $L_{i}$ and its meridian $\mu_{i}$. Let $\hat{R} \times[0,1]$ be a product neighborhood of $\widehat{R}$ in $M$ so that the corresponding interval orientation on $R \times[0,1]$ corresponds to the meridian orientation of $L_{1}$. Pick coordinates $\widehat{R}=$ $e^{2 \pi i \theta} \times[0,1]$, with $\theta \in[0,1]$, so that $e^{2 \pi i \theta} \times\{0\}, \theta \in[0,1]$, is the oriented $L_{1}$. Define the homeomorphism

$$
f_{n}: \hat{R} \times[0,1] \rightarrow \widehat{R} \times[0,1], \quad\left(e^{2 \pi i \theta}, s, t\right) \mapsto\left(e^{2 \pi i(\theta+n t)}, s, t\right) .
$$

Note that $f_{n}$ restricted to $\widehat{R} \times\{0,1\}$ is the identity. Assume that the knot $K$ in $M$ intersects $\widehat{R} \times[0,1]$ in $[0,1]$ fibers. Let $K^{n}$ be the knot in $M$ gotten by applying $f_{n}$ to $K \cap(\hat{R} \times[0,1])$ (and the identity on $K$ outside this region). We refer to $K^{n}$ as $K$ twisted $n$ times along $\hat{R}$. Furthermore, note that $f_{n}$ induces a homeomorphism

$$
h_{n}: M-\mathcal{N}\left(L_{1} \cup L_{2}\right) \rightarrow M-\mathcal{N}\left(L_{1} \cup L_{2}\right)
$$


by applying $f_{n}$ in $R \times[0,1]$ along with the identity outside this neighborhood. We refer to this homeomorphism $h_{n}$ of $M-\mathcal{N}\left(L_{1} \cup L_{2}\right)$ as $n$ Dehn-twists along the properly embedded annulus $R$. Note that $K^{n}$ only depends on the isotopy class of $K$ in the complement of $L_{1} \cup L_{2}$. Furthermore, one can check that $f_{n}, K^{n}, h_{n}$ are independent of the orientation chosen on $\hat{R}$.

For an annulus $\hat{R}$ and knot $K$ in $M$, we say the pair $(\hat{R}, K)$ is caught if some oriented surface $Q$ properly embedded in the exterior $X=M-\mathcal{N}(K \cup \partial \hat{R})$ intersects both components of $\partial \mathcal{N}(\partial \hat{R})$ in slopes (ie unoriented isotopy classes) different than the framing induced by $\hat{R}$ and with non-trivial homology on each of those components (Definition 2.1). Lemma 2.5 shows that it is often the case that $(\hat{R}, K)$ is caught.

When $H_{1} \cup_{\widehat{F}} H_{2}$ is a Heegaard splitting of $M$ and $J$ is a knot in $M$ we denote by $\mathfrak{b}_{\widehat{F}}(J)$ the bridge number of $J$ with respect to this splitting (see Section 2.3). Here we allow Heegaard splittings in a manifold with boundary given by a union of compression bodies (see Section 2.2). The distance between two simple closed curves on a 2-torus is the minimal geometric intersection number of the curves up to isotopy (Section 2.1). In Section 2.6, we prove the following Theorem 1.3 and its corollaries Corollary 1.1 above and Corollary 1.4 below.

Theorem 1.3 Let $M$ be a compact, orientable 3-manifold with (possibly empty) boundary and $K \cup L_{1} \cup L_{2}$ a link in $M$. Let $\widehat{R}$ be an annulus in $M$ with $\partial \widehat{R}=L_{1} \cup L_{2}$. Assume $(\hat{R}, K)$ in $M$ is caught by the surface $Q$ in $X=M-\mathcal{N}\left(K \cup L_{1} \cup L_{2}\right)$. Let $T_{K}, T_{1}, T_{2}$ be the components of $\partial X$ corresponding to $K, L_{1}, L_{2}$ respectively. Fixing an orientation on $M$, let $\mu_{i}$ be a meridian of $L_{i}$ on $T_{i}$ and $\lambda_{i}$ be a framing curve coming from $\hat{R}$. Express the first homology class of a component of $\partial Q$ on $T_{i}$ as $p_{i}\left[\mu_{i}\right]+q_{i}\left[\lambda_{i}\right]$. Let $\Delta_{K}$ be the distance on $T_{K}$ between a component of $\partial Q$ and a meridian of $K$ (setting $\Delta_{K}=0$ when $Q$ is disjoint from $K$ ). Let $K^{n}$ be $K$ twisted $n$ times along $\widehat{R}$ (Definition 1.2).

Let $H_{1} \cup \widehat{F} H_{2}$ be a genus- $g$ Heegaard splitting of $M$.

Then one of the following holds:

(1) $\hat{R}$ can be isotoped to lie in $\widehat{F}$.

(2) There is an essential annulus $A$ properly embedded in $X$ with a boundary component in each of $T_{1}$ and $T_{2}$, and furthermore the slope of $\partial A$ on each $T_{i}$ is neither that of the meridian of $\mathcal{N}\left(L_{i}\right)$ nor that of $\partial Q$.

(3) For each $n$,

$$
\mathfrak{b}_{\widehat{F}}\left(K^{n}\right) \geq \frac{\min \left(\left|q_{1}+n p_{1}\right|,\left|q_{2}-n p_{2}\right|\right) / \max (-36 \chi(Q), 6)-2 g+1}{2 \max \left(\Delta_{K}, 1\right)} .
$$


To be able to use the bound in Theorem 1.3(3), one needs to know that conclusions (1) and (2) do not hold. If (1) holds then $\left\{\mathfrak{b}_{\widehat{F}}\left(K^{n}\right)\right\}$ is a finite set. So assume (1) does not hold. If (2) holds and $\partial A$ is not isotopic to $\partial \hat{R}$ on $T_{1} \cup T_{2}$, then $A$ can be used as a catching surface for $(\hat{R}, K)$. Applying Theorem 1.3 with $Q=A$ will force conclusion (3) or exhibit a new annulus in $X$ whose boundary is isotopic to $\partial \widehat{R}$ on $T_{1} \cup T_{2}$ (see Lemma 2.14 and the proof of Corollary 1.1). The first gives a lower bound in $n$ on $\mathfrak{b}_{\widehat{F}}\left(K^{n}\right)$ in terms of the slopes of $\partial A$. On the other hand, if there is an annulus in $X$ whose boundary is isotopic on $T_{1} \cup T_{2}$ to $\partial \hat{R}$ then $\left\{\mathfrak{b}_{g}\left(K^{n}\right)\right\}$ will be finite (though $\left\{\mathfrak{b}_{\widehat{F}}\left(K^{n}\right)\right\}$ is not necessarily finite). Focusing on $\left\{\mathfrak{b}_{g}\left(K^{n}\right)\right\}$, Theorem 1.3 then leads to Corollary 1.1 above, for which a more detailed argument is given in Section 2.6.

Applying Corollary 1.1 to manifolds $M$ with small-genus Heegaard splittings, we have the following.

Corollary 1.4 Assume that $M$ is closed and orientable and let $K \cup L_{1} \cup L_{2}$ be a link in $M$. Let $\hat{R}$ be an annulus in $M$ with $\partial \hat{R}=L_{1} \cup L_{2}$, and let $R$ be the annulus $\hat{R} \cap\left(M-\mathcal{N}\left(L_{1} \cup L_{2}\right)\right)$ properly embedded in $M-\mathcal{N}\left(L_{1} \cup L_{2}\right)$. Assume that $(\hat{R}, K)$ in $M$ is caught. Assume there is no properly embedded, essential annulus $A$ in $X=M-\mathcal{N}\left(K \cup L_{1} \cup L_{2}\right)$ such that $\partial A \cap\left(T_{1} \cup T_{2}\right)$ is isotopic to $\partial R \cap\left(T_{1} \cup T_{2}\right)$ on $T_{1} \cup T_{2}$. Fixing an orientation on $M$, let $K^{n}$ be $K$ twisted $n$ times along $\hat{R}$.

If $M=S^{3}$ and $L_{1} \cup L_{2}$ is not the trivial link, then $\mathfrak{b}_{0}\left(K^{n}\right) \rightarrow \infty$ as $n \rightarrow \infty$.

If $M$ is a lens space and $L_{1} \cup L_{2}$ is not a lens space torus link, then $\mathfrak{b}_{1}\left(K^{n}\right) \rightarrow \infty$ as $n \rightarrow \infty$.

If $M$ has Heegaard genus at most 2, then either $\mathfrak{b}_{2}\left(K^{n}\right) \rightarrow \infty$ as $n \rightarrow \infty$ or one of the following holds:

(a) $L_{1}$ has tunnel number one in $M$ (or bounds a disk in $M$ ).

(b) $L_{1}$ is a cable of a tunnel number one knot in $M$ where the slope of the cabling annulus is that of $\partial R$.

(c) 0-surgery (as framed by $R$ ) on $L_{1}$ contains an essential torus.

As $L_{1}$ and $L_{2}$ are isotopic in $M$, if any of (a)-(c) holds for $L_{1}$, then it also holds for $L_{2}$.

Beginning with an annulus $\widehat{R}$ and banding $\partial \widehat{R}$ together in a sufficiently complicated manner, Osoinach [14] produced infinite families of distinct knots in $S^{3}$ for which the same integral surgery produces the same manifold $M$. The knots in such a family are related by twisting along $\hat{R}$, and the surgery-duals are related by twisting along an 
annulus in $M$. Teragaito [17] adapted this construction to develop an infinite family of distinct knots for which +4 -surgery produces the same small Seifert fiber space $M$. In Section 3, we apply Corollary 1.4 to prove the following.

Theorem 1.5 Let $\left\{K^{\prime n}\right\}$ be the Teragaito family of knots in $S^{3}$. For each $n$, let $K^{n} \subset M$ be the +4 -surgery-dual to $K^{\prime n}$ with respect to the Seifert framing on $K^{\prime n}$. Then $\mathfrak{b}_{0}\left(K^{\prime n}\right) \rightarrow \infty$ and $\mathfrak{b}_{2}\left(K^{n}\right) \rightarrow \infty$ as $n \rightarrow \infty$.

Remark 1.6 This is in sharp contrast to what occurs for non-integral surgeries. Corollary 1.1 of [2] shows that if a non-integral surgery on a hyperbolic knot in $S^{3}$ produces a small Seifert fiber space then the genus- 2 bridge number of the surgery-dual is at most 10975. Theorem 2.4 of [1] shows that if $p / q$-surgery, with $|q|>2$, on a hyperbolic knot in $S^{3}$ produces a manifold $M$ with Heegaard genus 2 , and $M$ contains no Dyck's surface, then the genus- 2 bridge number of the surgery-dual is at most 1 . To further contrast the results of [2] and [1], in Section 3 we generalize the Teragaito family to give other families of knots in the 3-sphere, where each knot in a family admits a surgery giving the same genus- 2 manifold $M$ and where the surgery-duals to that family have arbitrarily large genus- 2 bridge numbers in $M$ (Theorem 3.2). Generically these $M$ are hyperbolic manifolds, whereas for the Teragaito family $M$ is Seifert fibered. In Lemma 3.10 we show that infinitely many of these hyperbolic $M$ do not contain Dyck's surfaces, to support the contrast with [1, Theorem 2.4].

Remark 1.7 A conjecture of Berge says that if a knot $K^{\prime}$ in $S^{3}$ admits a Dehn surgery which is a lens space $M$ then the bridge number of the surgery-dual $K \subset M$ with respect to a minimal-genus Heegaard splitting of $M$ is one, ie $\mathfrak{b}_{1}(K)=1$. Thus Theorem 1.5 contrasts the expected picture for lens space and small Seifert fiber space surgeries on knots in $S^{3}$.

Remark 1.8 Question 3.1 of Mattman, Miyazaki and Motegi [12] asks: If an integral surgery on a hyperbolic knot in $S^{3}$ produces a small Seifert fibered space $M$, then does the dual knot embed in a genus-2 Heegaard surface for $M$ ? Teragaito showed that the dual knots to his examples answered this question in the negative; that the dual knots do not lie on a genus- 2 Heegaard surface. Theorem 1.5 shows that in fact these knots have arbitrarily large bridge number with respect to genus- 2 splittings of $M$.

Remark 1.9 Teragaito [17] also describes a related second infinite family of distinct knots for which +4 -surgery always produces a certain small Seifert fibered space. We conclude Section 3 by showing that the set of genus- 2 bridge numbers of the knots surgery-dual to Teragaito's second family is bounded (Theorem 3.8). 
Acknowledgements This work was partially supported by grant number 209184 to Kenneth L Baker from the Simons Foundation and by NSF grant DMS 0906276 to Cameron Gordon. The authors would like to thank Sean Bowman for helpful conversations.

\section{Bounding bridge numbers}

\subsection{Slopes and surgeries}

A slope is an isotopy class of unoriented simple closed curves on a torus. We also say the slope of a collection of isotopic simple closed curves on a torus is the slope of any individual curve. The distance of two slopes $\alpha, \beta$ is the minimal geometric intersection number among curves representing these classes and is denoted $\Delta(\alpha, \beta)$. Let $\mu$ be the meridional slope of a knot $K$ in a manifold $M$. Dehn surgery on a $K$ along a slope $\gamma$ is integral or longitudinal if $\Delta(\mu, \gamma)=1$, non-integral if $\Delta(\mu, \gamma)>1$, and trivial if $\Delta(\mu, \gamma)=0$. In the surgered manifold, the core of the attached solid torus is the surgery-dual of $K$.

\subsection{Spines and core curves of handlebodies and compression bodies}

A spine $\Gamma$ of a handlebody $H$ is a properly embedded graph such that $H-\Gamma \cong$ $\partial H \times(-\infty, 0]$. For a compression body $H$ with $\partial H$ partitioned as $\partial_{+} H \cup \partial_{-} H$ with $\partial_{+} H$ connected, a spine $\Gamma$ of $H$ is a properly embedded graph disjoint from $\partial_{+} H$ such that $H-\left(\Gamma \cup \partial_{-} H\right) \cong \partial_{+} H \times(-\infty, 0]$.

An embedded closed curve $C$ in the interior of a handlebody or compression body $H$ is a core curve (or just core) if there is a spine $\Gamma$ of $H$ such that $C$ may be isotoped into $\Gamma$. For a core $C$ of $H, H-\mathcal{N}(C)$ is a compression body. When $H$ is a solid torus, we usually speak of the core since all core curves are isotopic.

\subsection{Heegaard splittings, thin position, and bridge position}

In this paper, a Heegaard splitting will always be a 2-sided Heegaard splitting. In particular, a Heegaard splitting of a 3-manifold with boundary, $Y$, is the writing of $Y$ as the union of two compression bodies $H_{1}$ and $H_{2}$ along their boundary components $\partial_{+} H_{1}$ and $\partial_{+} H_{2}$. The shared boundary of these compression bodies is the Heegaard surface of the splitting. Given such a Heegaard surface $S$ of $Y$ there is a product $S \times \mathbb{R} \subset Y$ so that $S=S \times\{0\}$ and the complement of the product is the union of a pair of spines of the two compression bodies along with $\partial Y$. This defines a height 
function on the complement in $Y$ of $\partial Y$ and the spines of the compression bodies. Consider all the circles $C$ embedded in the product that are Morse with respect to the height function and represent the knot type of a particular knot $J$. The following terms are all understood to be taken with respect to the Heegaard splitting.

Following [5] (see also [15]), the width of an embedded circle $C$ is the sum of the number of intersections $\left|C \cap S \times\left\{y_{i}\right\}\right|$ where one regular value $y_{i}$ is chosen between each pair of consecutive critical values of $C$. The width of a knot $J$ is the minimum width of all such embeddings. An embedding realizing the width of $J$ is a thin position of $J$, and $J$ is said to be thin.

The minimal number of local maxima among Morse embeddings is the bridge number of $J$, and denoted $\mathfrak{b}_{S}(J)$, or, if $S$ is understood, $\mathfrak{b}(J)$. An embedding realizing the bridge number of $J$ may be ambiently isotoped so that all local maxima lie above all local minima, without introducing any more extrema. The resulting embedding is a bridge position of $J$, and $J$ is said to be bridge. For a fixed genus $g$ of Heegaard splittings of $Y$, let $\mathfrak{b}_{g}(J)$ be the minimum bridge number of $J$ among genus- $g$ Heegaard splittings of $Y$.

By definition, bridge numbers are positive. It is common to say that if $J$ can be isotoped to lie on $S$ then $\mathfrak{b}_{S}(J)=0$. We will not use that terminology in this paper; for such a knot we take $\mathfrak{b}_{S}(J)=1$. That is, bridge and thin presentations of a knot or link will always be Morse with respect to the given height function.

The definition of thin position extends to links. If $K$ is a sublink of the link $J$, then a $K$-thin position of $J$ (with respect to the Heegaard splitting) is a thinnest (least width) position of $J$ among those that restrict to a thin position of $K$.

\section{$2.4 Q$ catches $(\hat{\boldsymbol{R}}, K)$}

Let $\widehat{R}$ be an annulus embedded in the interior of an orientable 3-manifold $M$ with $\partial \widehat{R}=L_{1} \cup L_{2}$. Let $K$ be a knot in $M$ disjoint from $L_{1} \cup L_{2}$ and transverse to $\widehat{R}$. Write $\mathcal{L}=K \cup L_{1} \cup L_{2}$, let $X=M-\mathcal{N}(\mathcal{L})$ be the exterior of the link $\mathcal{L}$, and set $R=\hat{R} \cap\left(M-\mathcal{N}\left(L_{1} \cup L_{2}\right)\right)$. Let $T_{K}, T_{1}, T_{2}$ be the torus components of $\partial X$ corresponding to $K, L_{1}, L_{2}$, respectively.

Definition 2.1 Let $Q$ be an oriented (possibly disconnected) surface, properly embedded in $X$ with no disk components or closed components. Furthermore, assume that if $Q$ has annular components then $Q$ is a single annulus. We say that $Q$ catches the pair $(\widehat{R}, K)$ if

- $\partial Q \cap T_{i}$ is a non-empty collection of coherently oriented parallel curves on $T_{i}$ for each $i \in\{1,2\}$; and

- $\partial Q$ intersects both $T_{1}$ and $T_{2}$ in slopes different than $\partial R$. 
We say the pair $(\widehat{R}, K)$ is caught if it has a catching surface.

Remark 2.2 Let $Q$ be a catching surface for $(K, \widehat{R})$. By discarding components, we may assume that each component of $Q$ has some boundary component in $T_{1} \cup T_{2}$. We may in fact assume that $Q$ has at most two components, and when $Q$ has two then one of these components is disjoint from $T_{1}$ and the other disjoint from $T_{2}$. Note that if there were a disk in $X$ with boundary on $T_{1} \cup T_{2}$ then its boundary would have to be parallel to a component of the boundary of $\hat{R}$. If there were an annulus in $X$ with only one boundary component on $T_{1} \cup T_{2}$, then the existence of $\hat{R}$ implies that the other must be on $T_{K}$. If there were two such annuli, one with a boundary component on $T_{1}$, the other with a boundary component on $T_{2}$, these annuli could be used to construct a single annulus with boundary in $T_{1} \cup T_{2}$.

Remark 2.3 When $M$ is closed, the half lives/half dies lemma says that the image of $\partial_{*}: H_{2}(X, \partial X) \rightarrow H_{1}(\partial X)$ has half the rank of $H_{1}(\partial X)$; see eg [7, Lemma 3.5]. This guarantees that there is a $Q$ such that

- the components of $\partial Q$ are coherently oriented parallel curves on the components of $\partial X$, and

- $\quad[\partial Q]$ is not a multiple of $[\partial R]$ in $H_{1}(\partial X)$.

Definition 2.4 Given a knot $K$ in a closed 3-manifold $M$, we say an orientable surface $\Sigma$ with boundary that is properly embedded in $M-\mathcal{N}(K)$ is a generalized Seifert surface for $K$ if $\partial \Sigma$ is a collection of coherently oriented parallel curves on $\partial \mathcal{N}(K)$ once $\Sigma$ is oriented. By the half lives/half dies lemma, every such knot $K$ has a generalized Seifert surface. Note that the boundary of a generalized Seifert surface may be a collection of meridional curves.

Lemma 2.5 A pair $(\hat{R}, K)$ in a closed 3-manifold $M$ is not caught if and only if $L_{1}$ has a generalized Seifert surface disjoint from $L_{2}$ and $K$ has a generalized Seifert surface disjoint from either $L_{1}$ or $L_{2}$.

Proof If $L_{1}$ does not have a generalized Seifert surface disjoint from $L_{2}$, then there exists one, say $\Sigma_{1}$, which is transverse to $L_{2}$ and $K$ and such that, when oriented, $\Sigma_{1} \cap \partial \mathcal{N}\left(L_{2}\right)$ is a non-empty collection of coherently oriented meridians of $L_{2}$. Since the boundary slope of $\Sigma_{1}$ on $\partial \mathcal{N}\left(L_{1}\right)$ is necessarily different than that of $R$, $Q=\Sigma_{1} \cap X$ catches $(\widehat{R}, K)$.

If $K$ does not have a generalized Seifert surface disjoint from either $L_{1}$ or $L_{2}$, then there exists one, say $\Sigma_{K}$, which is transverse to $L_{1} \cup L_{2}$ and such that, when oriented, $\Sigma_{K} \cap \partial \mathcal{N}\left(L_{i}\right)$ is a non-empty collection of coherently oriented meridians of $L_{i}$, $i=1,2$. Thus $Q=\Sigma_{K} \cap X$ catches $(\hat{R}, K)$. 
Now assume $\Sigma_{1}$ is a generalized Seifert surface for $L_{1}$ that is disjoint from $L_{2}$ and transversely intersects $K$ and $\Sigma_{K}$ is a generalized Seifert surface for $K$ that is disjoint from $L_{1} \cup L_{2}$. Set $\Sigma_{1}^{\prime}=\Sigma_{1} \cap X$. Recall that $\widehat{R}$ is an annulus with boundary $L_{1} \cup L_{2}$ that $K$ transversely intersects. Let $\lambda_{2}=R \cap T_{2}$.

Observe that $\left[\partial \Sigma_{1}^{\prime}\right],\left[\partial \Sigma_{K}\right]$, and $[\partial R]$ together generate a rank-3 subgroup of $H_{1}(\partial X)$ whose intersection with $H_{1}\left(T_{2}\right)$ is generated by $\left[\lambda_{2}\right]$. If a surface $Q \subset X$ were to catch $(\hat{R}, K)$ then together $\left[Q \cap T_{2}\right]$ and $\left[\lambda_{2}\right]$ would generate a rank-2 subgroup of $H_{1}\left(T_{2}\right)$. But then $[\partial Q]$ with $\left[\partial \Sigma_{1}^{\prime}\right],\left[\partial \Sigma_{K}\right]$, and $[\partial R]$ would generate a subgroup of $H_{1}(\partial X)$ of rank at least 4 . This contradicts the half lives/half dies lemma. Hence $(\widehat{R}, K)$ cannot be caught.

Remark 2.6 Notice that $L_{1}$ has a generalized Seifert surface disjoint from $L_{2}$ if and only if its boundary slope on $\partial \mathcal{N}\left(L_{1}\right)$ agrees with the boundary slope of $R$. If $\Sigma_{1}$ is a generalized Seifert surface for $L_{1}$ that is disjoint from $L_{2}$, then we may use copies of $\widehat{R}$ to extend $\Sigma_{1}$ to a generalized Seifert surface for $L_{2}$ which an isotopy will make disjoint from $L_{1}$. Hence $L_{1}$ has a generalized Seifert surface disjoint from $L_{2}$ if and only if $L_{2}$ has a generalized Seifert surface disjoint from $L_{1}$.

\subsection{Combinatorics}

Let $\mathcal{L}=K \cup L_{1} \cup L_{2}$ be a link in a compact, orientable 3-manifold $M$ (possibly with boundary) and $X=M-\mathcal{N}(\mathcal{L})$ its exterior. Let $T_{K}, T_{1}, T_{2}$ be the torus components of $\partial X$ corresponding to $K, L_{1}, L_{2}$. In this section we assume that $Q$ is a properly embedded, orientable surface in $X$ such that $\partial Q \cap T_{i}$ is non-empty and non-meridional for $i \in\{1,2\}$.

Recall from Section 2.3 that a genus- $g$ Heegaard splitting $H_{1} \cup_{\widehat{F}} H_{2}$ of $M$ gives a height function in the complement of the compression body spines and $\partial M$. With $\mathcal{L}$ in $K$-thin position with respect to this Heegaard splitting, isotope $Q$ so that, in a neighborhood of any local maximum or minimum of $\mathcal{L}, Q$ is below or above $\mathcal{L}$, respectively, and $\partial Q$ is transverse to the foliation by level curves on the rest of $T_{1} \cup T_{2} \cup T_{K}$ (if the components of $\partial Q \cap T_{K}$ are meridional, we take each of these components to be level). Near components of $\partial M$ we may take $Q$ transverse to the level surfaces. We take $Q$ transverse to the compression body spines. We may then further isotope $Q$, away from $\partial Q$ and away from the compression body spines, to be transverse to the level surfaces except at a finite number of points which all occur at distinct levels, distinct from the extrema of $\mathcal{L}$ too.

Given any level surface $\widehat{P}$ of this height function away from a critical level of $Q$, set $P=X \cap \widehat{P}$. By the above isotopy of $Q, \partial Q$ intersects $\partial P$ minimally on $\partial X$. For 

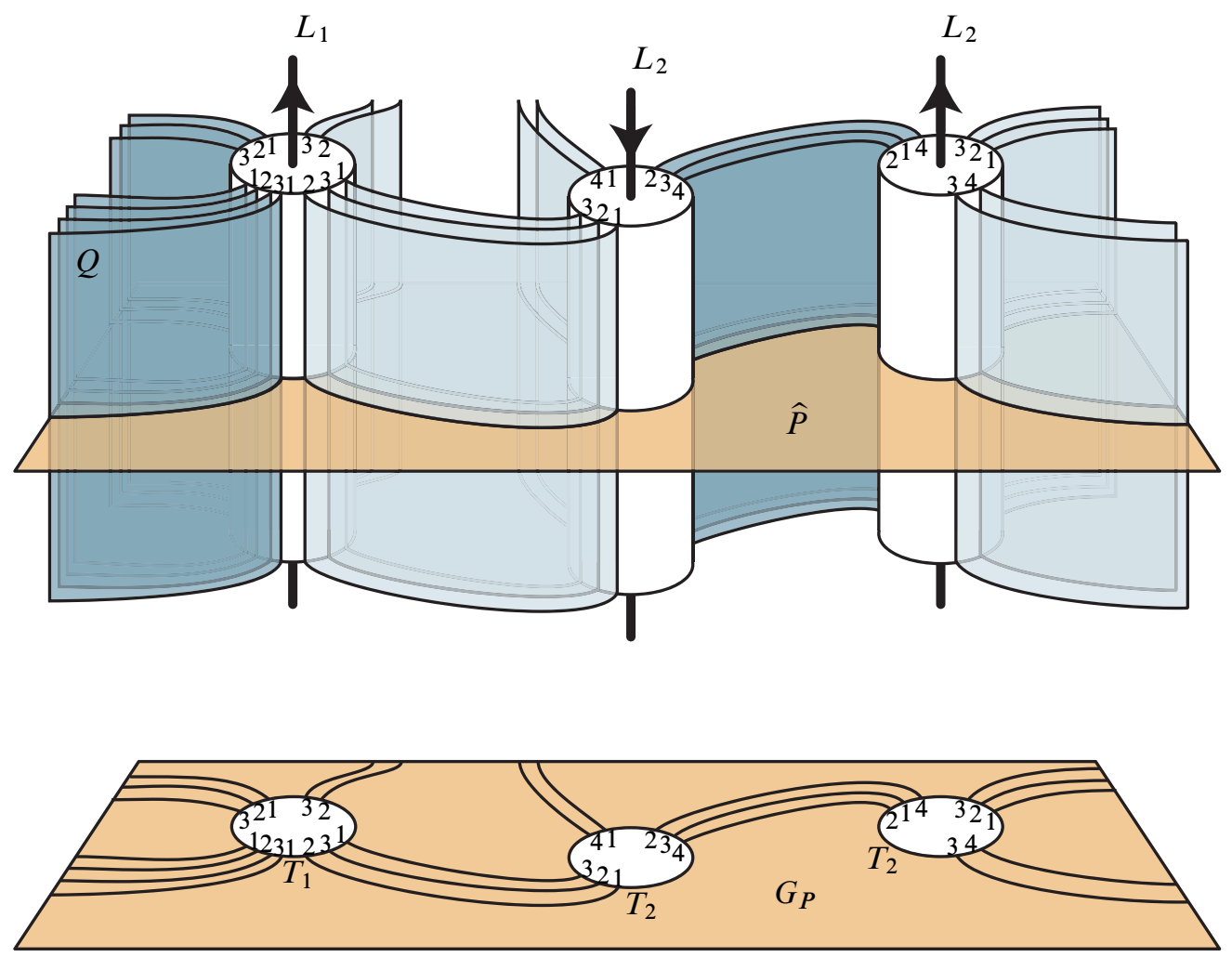

Figure 1: An example of a portion of the graph $G_{P}$ arising from the intersection of $Q$ with $\widehat{P}$ and, say, $\left|\partial Q \cap T_{1}\right|=3$ with $\Delta_{1}=4$ and $\left|\partial Q \cap T_{2}\right|=4$ with $\Delta_{2}=2$. Vertices of $G_{P}$ are marked according to their corresponding component of $\partial X$.

such a level surface $\widehat{P}$, form the corresponding pair of labeled fat vertexed graphs of intersection $G_{P}$ and $G_{Q}$, as follows. Define $\widehat{Q}$ to be $Q$ with disks attached along the components of $\partial Q \cap\left(T_{K} \cup T_{1} \cup T_{2}\right)$ when $\partial Q \cap T_{K}$ are not meridians of $K$, and along the components of $\partial Q \cap\left(T_{1} \cup T_{2}\right)$ when $\partial Q \cap T_{K}$ is meridional. Then $G_{P}$ and $G_{Q}$ are the graphs on the surfaces $\widehat{P}$ and $\widehat{Q}$, respectively, consisting of the fat vertices that are the disks $\mathcal{N}(\mathcal{L}) \cap \widehat{P}$ on $\widehat{P}$ and the disks that cap off $\partial Q$ in $\widehat{Q}$, and the edges that are the arcs of $P \cap Q$. Note that $\mathcal{N}(K) \cap \widehat{P}$ become vertices of valence 0 when $\partial Q \cap T_{K}$ is meridional on $K$. Label the endpoint of an edge in one graph with the vertex of the other graph whose boundary contains the endpoint. Figure 1 gives an example of how the graph $G_{P}$ arises.

Fix orientations of $\widehat{P}$ and $\hat{Q}$. Two vertices on the same graph and on the same component of $\partial X$ are parallel if their corresponding oriented components of $\partial P$ or $\partial Q$ 
are coherently oriented on $\partial X$; they are anti-parallel otherwise. The orientability of $P, Q$ and $X$ gives the parity rule: an edge connecting parallel vertices on one graph must connect anti-parallel vertices on the other graph.

Let $\Delta_{K}, \Delta_{1}, \Delta_{2}$ be the distances between the slopes of $\partial P$ and $\partial Q$ on $T_{K}, T_{1}, T_{2}$, respectively. If $\partial Q$ or $\partial P$ is disjoint from $T_{K}$, we set $\Delta_{K}=0$. Note that if $\partial Q \cap T_{K}$ is meridional on $K$, then $\Delta_{K}=0$. By assumption, $\Delta_{1}, \Delta_{2}$ are non-zero. Set

$$
m_{K}=|\widehat{P} \cap K| \leq 2 \mathfrak{b}_{\widehat{F}}(K), \quad m_{1}=\left|\widehat{P} \cap L_{1}\right|, \quad m_{2}=\left|\widehat{P} \cap L_{2}\right| ;
$$

these are the numbers of vertices in $G_{P}$ corresponding to $K, L_{1}, L_{2}$, respectively. Number the components of $\partial P$ on a component of $\partial X$ in sequence $1, \ldots, m_{i}$.

Let $V_{K}, V_{1}, V_{2}$ be the sets of vertices of $G_{Q}$ corresponding to $K, L_{1}, L_{2}$, respectively ( $V_{K}$ is empty when $\partial Q \cap T_{K}$ is meridional). The vertices in each of these sets may also be numbered in the order they appear around their component of $\partial X$. Observe that a vertex $v \in V_{i}$ has valence $m_{i} \Delta_{i}$ for $i=K, 1,2$; in particular, the $m_{i}$ labels of corresponding vertices in $G_{P}$ appear in order $\Delta_{i}$ times around $v$.

Note that $P \cap Q$ has an arc component which is boundary-parallel in $Q$ if and only if $G_{Q}$ has a monogon face, ie a face bounded by a fat vertex and single edge of $G_{Q}$.

Lemma 2.7 Given a Heegaard splitting $\mathrm{H}_{1} \cup_{\widehat{F}} \mathrm{H}_{2}$ of $M$, there is a $K$-thin presentation for $\mathcal{L}$ such that one of the following holds:

(A) There is a level surface $\widehat{P}$ transverse to $Q$ and with non-empty intersection with $L_{1} \cup L_{2}$ such that there is no monogon of $G_{Q}$ at any vertex of $V_{1} \cup V_{2}$. If the components of $\partial Q \cap T_{K}$ are meridional on $K$, then $\widehat{P}$ is disjoint from $\partial Q \cap T_{K}$.

(B) There is a level surface $\widehat{P}$ transverse to $Q$ such that for some choice of $\{i, j\}=$ $\{1,2\}, m_{i} \geq m_{j}=2$ and there is no monogon of $G_{Q}$ at any vertex of $V_{i}$. If the components of $\partial Q \cap T_{K}$ are meridional on $K$, then $\widehat{P}$ is disjoint from $\partial Q \cap T_{K}$.

(C) $L_{1} \cup L_{2}$ can be isotoped disjointly from $K$ (keeping $K$ fixed) so that $L_{1}$ and $L_{2}$ lie on disjoint copies of $\hat{F}$.

Proof Take a $K$-thin presentation of $\mathcal{L}$ with respect to the given splitting. In this Morse presentation of $\mathcal{L}$, let $I$ be a middle slab, ie an interval of level surfaces without critical points of $\mathcal{L}$ in its interior whose upper and lower levels contain a maximum and minimum of $\mathcal{L}$, respectively.

We choose $I$ so that the intersection of $L_{1} \cup L_{2}$ with any level surface in $I$ is nonempty. If there is a level surface $\widehat{P}$ in $I$, transverse to $Q$, giving rise to no monogons in $G_{Q}$ at each of $V_{1}$ and $V_{2}$, then (A) is satisfied and we are done. (Possibly $\widehat{P}$ is disjoint from $K$ or one, but not both, of $L_{1}, L_{2}$.) 
So assume for each transverse level surface in this slab $I$ there is a high or low disk in $G_{Q}$ associated to $L_{1} \cup L_{2}$. (A monogon of $G_{Q}$ is a high or low disk if a collar of its boundary lies above or below the level surface $\widehat{P}$ in $M$, respectively.) Apply Gabai's argument (in [5, Lemma 4.4]) to the high and low disks coming from these monogons of $G_{Q}$. Note that near the maximum of $I$ such a disk must be high, and near the minimum it must be low. Gabai's argument in this context shows that there must be a level surface $\widehat{P}$ that intersects some $L_{j}$ twice, for some $j \in\{1,2\}$, and gives rise to high and low disks in $G_{Q}$ guiding $L_{j}$ onto $\widehat{P}$ disjointly from the other two components of $\mathcal{L}$. Then $Q$ cannot also give rise to either a high or low disk at $\widehat{P}$ for another component of $\mathcal{L}$ since otherwise $\mathcal{L}$ could be thinned without increasing the width of $K$. Taking $\{i, j\}=\{1,2\}, \widehat{P}$ satisfies (B) unless $\widehat{P}$ is disjoint from $L_{i}$, which we now assume.

To the side of $\widehat{P}$ containing $L_{i}$ we may find a new middle slab such that each level surface intersects $L_{i}$ but is disjoint from $L_{j}$. Otherwise by isotoping $L_{j}$ onto $\widehat{P}$ we could thin. Now apply the same argument. Either we find a level surface satisfying (A) or $L_{i}$ can be isotoped disjointly from $K \cup L_{j}$ onto a level surface $\hat{P}^{\prime}$ in this slab. Therefore, assuming (A) does not occur for a level surface in this new middle slab, we may isotope $L_{1} \cup L_{2}$ disjointly from $K$ onto distinct level surfaces $\widehat{P}$ and $\hat{P}^{\prime}$ so that $L_{1}$ lies in one and $L_{2}$ in the other, giving conclusion (C).

Lemma 2.8 Let $M$ be an orientable, compact 3-manifold and let $K \cup L_{1} \cup L_{2} \subset M$ be a link. Let $X=M-\mathcal{N}\left(K \cup L_{1} \cup L_{2}\right)$ and $T_{K}, T_{1}, T_{2}$ be the components of $\partial X$ corresponding to $K, L_{1}, L_{2}$. Let $Q \subset X$ be a properly embedded, oriented surface such that $T_{i} \cap \partial Q$ is a non-empty collection of coherently oriented curves on $T_{i}$ for each $i \in\{1,2\}$. Let $H_{1} \cup \widehat{F} H_{2}$ be a genus- $g$ Heegaard splitting of $M$. Assume that $L_{1} \cup L_{2}$ cannot be isotoped so that $L_{1}$ and $L_{2}$ lie on disjoint copies of $\hat{F}$. Let $\Delta_{K}, \Delta_{1}, \Delta_{2}$ be the distance between the slopes of $\partial Q$ and the meridian slopes of $K, L_{1}, L_{2}$ on the $T_{K}, T_{1}, T_{2}$. If

$$
\min \left(\Delta_{1}, \Delta_{2}\right)>\max (-36 \chi(Q), 6)\left(2 \mathfrak{b}_{\widehat{F}}(K) \max \left(\Delta_{K}, 1\right)+2 g-1\right)
$$

(where $\Delta_{K}=0$ includes the case that $\partial Q$ is disjoint from $\partial \mathcal{N}(K)$ ) then either

(a) there exists a Möbius band in $X$ whose boundary is a meridian in $T_{1}$ or $T_{2}$; or

(b) there exists an annulus in $X$ with one boundary component essential on $T_{1}$ and the other essential on $T_{2}$, and furthermore the slope of this annulus on $T_{i}$ is neither meridional nor that of $\partial Q \cap T_{i}$, for each $i \in\{1,2\}$.

Proof Recall that when $\Delta_{K}=0$ the components of $\partial Q \cap T_{K}$, if non-empty, are included in the boundary of $\hat{Q}$ (the abstract surface in which $G_{Q}$ sits) and $V_{K}$ is empty. Also note that by convention $\mathfrak{b}_{\widehat{F}}(K)>0$. 
Applying Lemma 2.7 to the given $\mathcal{L}=K \cup L_{1} \cup L_{2}, Q$, and Heegaard splitting, gives a level surface $\widehat{P}$ of the splitting for which we assume that conclusion (A) or (B) holds. Then $m_{i} \geq m_{j}$ and, say, $i=1$ so that $G_{Q}$ has no monogons based at a vertex of $V_{1}$.

Let $G_{Q}\left(V_{1}\right)$ be the subgraph in a subsurface of $G_{Q}$ consisting of all edges of $G_{Q}$ that are incident to $V_{1}$ and all the vertices of $G_{Q}$ to which these edges are incident. We think of $G_{Q}\left(V_{1}\right)$ as a graph in the surface gotten by attaching disks to $Q$ along those components of $\partial Q$ corresponding to vertices of $G_{Q}\left(V_{1}\right)$ (thus if a vertex of $G_{Q}$ is not connected by edges to $V_{1}$ then it will give rise to a boundary component of $G_{Q}\left(V_{1}\right)$ ). Let $\widetilde{G}_{Q}\left(V_{1}\right)$ be the reduced graph obtained from $G_{Q}\left(V_{1}\right)$ by amalgamating parallel edges. The graphs $G_{Q}, G_{Q}\left(V_{1}\right)$ and $\widetilde{G}_{Q}\left(V_{1}\right)$ are illustrated in Figure 2.

Claim 2.9 Assume $Q$ is an orientable surface with no disk components and such that each component has non-empty boundary. Furthermore assume that if $Q$ has annular components then $Q$ is a single annulus. Let $E$ be a collection of disjoint, properly embedded arcs in $Q$ such that no arc is parallel to the boundary and no two arcs are parallel to each other. Then $|E| \leq \max (-3 \chi(Q), 1)$.

Proof If $Q$ is an annulus then $|E| \leq 1$, verifying the inequality. So assume no component of $Q$ is an annulus. Since no arc of $E$ is boundary parallel and no two are parallel, $E$ can be completed to an ideal triangulation of (the interior of) $Q$ by adding more edges between the components of $\partial Q$ as needed. If $E^{\prime}$ is the resulting collection of edges and $F$ is the collection of ideal triangles, then we have both $3|F|=2\left|E^{\prime}\right|$ and $\chi(Q)=-\left|E^{\prime}\right|+|F|$. Thus $|E| \leq\left|E^{\prime}\right|=-3 \chi(Q)$. This gives the claim.

Since each vertex of $V_{1}$ has valence $m_{1} \Delta_{1}$, Claim 2.9 applied to $\widetilde{G}_{Q}\left(V_{1}\right)$ shows that there must be at least $m_{1} \Delta_{1} / \max (-6 \chi(Q), 1)$ mutually parallel edges of $G_{Q}\left(V_{1}\right)$. Let $\mathcal{E}$ be one of these sets of edges. Since the valence of a vertex of $V_{K}$ is

$$
m_{K} \Delta_{K} \leq 2 \mathfrak{b}_{\widehat{F}}(K) \Delta_{K}
$$

(the presentation is $K$-thin) which is in turn less than

$$
m_{1} \Delta_{1} / \max (-6 \chi(Q), 1)
$$

by hypothesis, the edges in $\mathcal{E}$ cannot have an endpoint on a vertex of $V_{K}$ in $G_{Q}$. Therefore the edges in $\mathcal{E}$ either (a) join two vertices of $V_{1}$ (perhaps the same vertex) or (b) join a vertex of $V_{1}$ to a vertex of $V_{2}$ (note that this must be the case if $Q$ is an annulus).

Now we show that there is a pair of edges of $\mathcal{E}$ bounding a disk on $\widehat{P}-\mathcal{N}(K)$. Let $G_{P}(\mathcal{E})$ be the subgraph of $G_{P}$ on $\widehat{P}-\mathcal{N}(K)$ consisting of the edges in $\mathcal{E}$ and the 

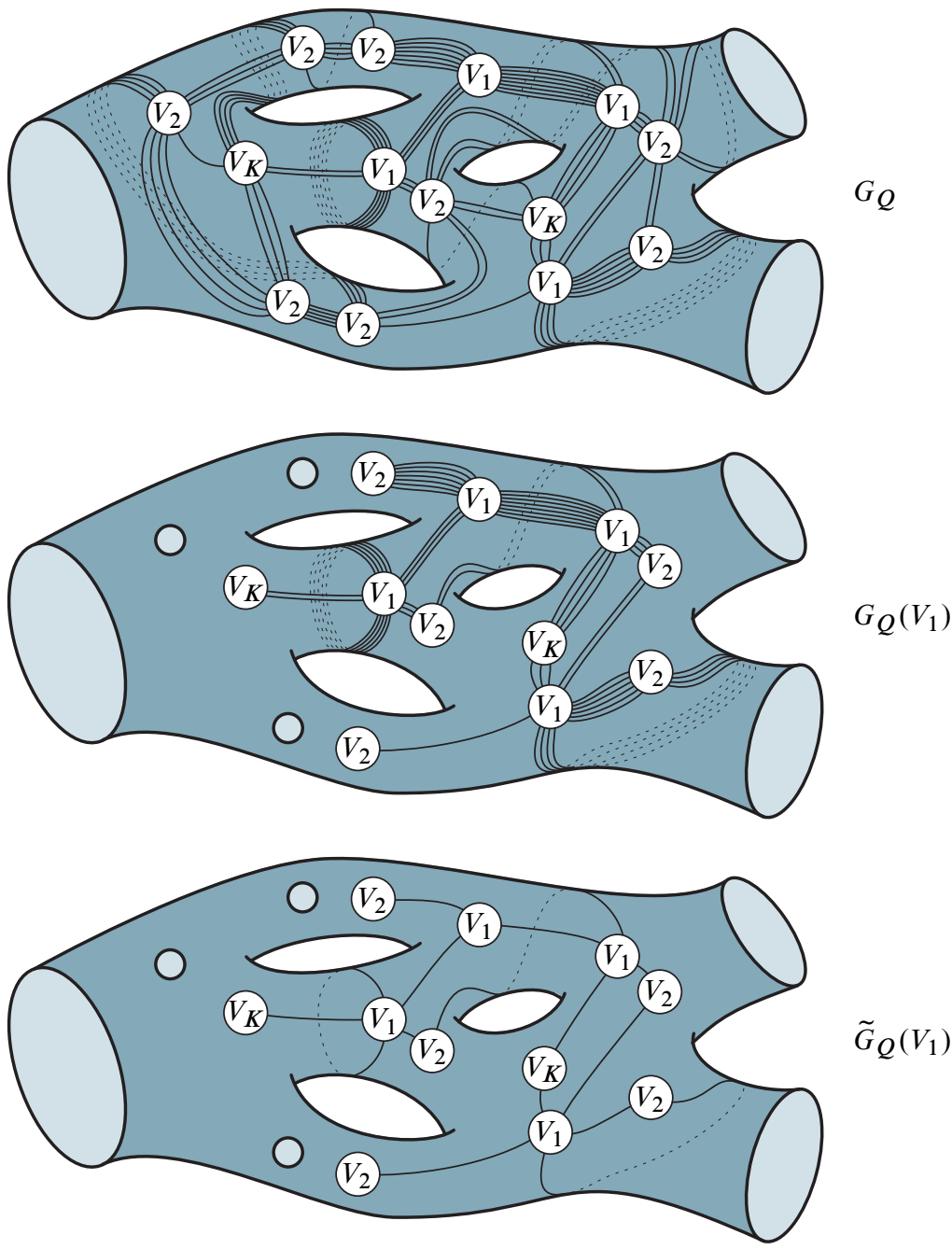

Figure 2: An example of the graphs $G_{Q}, G_{Q}\left(V_{1}\right)$, and $\widetilde{G}_{Q}\left(V_{1}\right)$. Vertices are marked according to which of the sets $V_{1}, V_{2}, V_{K}$ they belong.

vertices from $\hat{P} \cap\left(L_{1} \cup L_{2}\right)$ to which these edges are incident. For case (a), these vertices are all the $m_{1}$ vertices of $\hat{P} \cap L_{1}\left(\min \left(\Delta_{1}, \Delta_{2}\right)>\max (-6 \chi(Q), 1)\right)$. For case (b), notice that though the edges of $\mathcal{E}$ are parallel in $G_{Q}\left(V_{1}\right)$, in $G_{Q}$ these edges may have monogons interspersed between them at the $V_{2}$ vertex. However, if there are such monogons then we are under conclusion (B) of Lemma 2.7. Then $m_{2}=2$ and each of the two vertices of $\hat{P} \cap L_{2}$ appears $|\mathcal{E}| / 2$ times as a label at the $V_{2}$ end of $\mathcal{E}$. Whether we are working under conclusion (A) or (B) of Lemma 2.7 then, the hypotheses $\min \left(\Delta_{1}, \Delta_{2}\right)>\max (-6 \chi(Q), 1)$ and $m_{1} \geq m_{2}$ tell us that in case (b) 
the vertices of $G_{P}(\mathcal{E})$ are all the $m_{1}$ vertices of $\hat{P} \cap L_{1}$ with all the $m_{2}$ vertices of $\hat{P} \cap L_{2}$. In both cases (a) and (b), the vertices of $G_{P}(\mathcal{E})$ have valence at least $\Delta_{1} / \max (-6 \chi(Q), 1)$. (Each label of $G_{Q}$ at the vertices of $V_{1}$ or $V_{2}$ appears at least this many times at the endpoints of $\mathcal{E}$. For (b) we use that $m_{1} \geq m_{2}$.)

Claim 2.10 Let $G$ be a graph in a surface $P$ with $\chi(P)=k$. If $G$ has no monogons and each vertex has valence greater than $6 \max (1-k, 1)$ then $G$ has parallel edges.

Proof Assume there are no parallel edges in $G$. Then we may add edges to $G$ so that all faces are either $m$-gons with $m \geq 3$ or annuli with one boundary component being a component of $\partial P$ and the other consisting of a single edge and vertex of $G$. We may then count $\chi(P)$ as $V-E+F=k$, where $V, E, F$ are the numbers of vertices, edges and disk faces. Because every edge is on the boundary of the faces (including the annuli) twice, $2 E \geq 3 F+|\partial P|$. Let $C=6 \max (1-k, 1)$. The valence assumption implies $C V<2 E$ and thus both that $V<2 E / C$ and $C / 2<E$.

Therefore

$$
k=V-E+F<2 E / C-E+2 E / 3-|\partial P| / 3 .
$$

Hence $C k<E(2-C / 3)-|\partial P| C / 3$. Then, since $C \geq 6, k<0$. That is, $C=6(1-k)$. Thus

$$
3(1-k)>(1-1 / k)|\partial P|+E \geq E .
$$

This contradicts that $C / 2<E$.

Remark 2.11 When $k>0$ or $|\partial P| \neq 0$, this proof shows that if $G$ has no monogons and each vertex has valence at least $6 \max (1-k, 1)$ then $G$ has parallel edges. Change the strict inequalities in the last four lines to $\leq, \geq$. We conclude that $k \leq 0$ and $3(1-k) \geq(1-1 / k)|\partial P|+E>E$, the latter contradicting that $C / 2 \leq E$. In the application below, that $|\partial P|=0$ means that $K$ is disjoint from the level surface $\widehat{P}$.

Observe that $G_{P}(\mathcal{E})$ has no monogons: in case (a) by the parity rule due to the coherency of orientations of $\partial Q$ on the components of $\partial \mathcal{N}\left(L_{1} \cup L_{2}\right) \subset \partial X$, and in case (b) due to the endpoints of the edges being on vertices coming from different components of $L_{1} \cup L_{2}$. Note that in case (a) the vertices of $V_{2}$ are forgotten, so two edges that are parallel in $G_{P}(\mathcal{E})$ may not be parallel in $G_{P}$. Also each vertex of $G_{P}(\mathcal{E})$ has valence at least

$$
\begin{aligned}
\frac{\min \left(\Delta_{1}, \Delta_{2}\right)}{\max (-6 \chi(Q), 1)} & >6\left(2 \mathfrak{b}_{\widehat{F}}(K) \max \left(\Delta_{K}, 1\right)+2 g-1\right) \\
& \geq 6 \max (1-\chi(\widehat{P}-\mathcal{N}(K)), 1)
\end{aligned}
$$


because $\widehat{P}$ has genus $g,|\widehat{P} \cap K| \leq 2 \mathfrak{b}_{\widehat{F}}(K)$. Therefore Claim 2.10 implies that $G_{P}(\mathcal{E})$ has parallel edges. Hence there is a pair of edges $e, e^{\prime} \in \mathcal{E}$ that bound a disk $D_{Q}$ in $G_{Q}$ and a disk $D_{P}$ in $G_{P}(\mathcal{E})$. We may assume $D_{Q} \cap D_{P}=e \cup e^{\prime}$.

In case (a), $D_{Q} \cup D_{P}$ is a Möbius band in $M-\mathcal{N}\left(K \cup L_{1}\right)$ with boundary on $T_{1}$ that is a meridian. This follows from the proof of [6, Lemma 2.1]. To see that the boundary is a meridian, one notes that its slope is the same as the slope of $\partial P$ since the rectangle $D_{P}$ connects anti-parallel vertices in $P\left(D_{Q}\right.$ connects parallel vertices in $Q$ ).

In case (b), $D_{Q} \cup D_{P}$ is an annulus in $X$ with a boundary component on each of $T_{1}$ and $T_{2}$. Each boundary component of this annulus must intersect a component of $\partial P$ and of $\partial Q$ algebraically a non-zero number of times on $T_{1} \cup T_{2}$. Thus a boundary component of this annulus is essential and isotopic to neither a component of $\partial P$, a meridian, nor $\partial Q$. This is conclusion (b) of the lemma.

To finish the proof we need to show that the Möbius band of case (a) can be taken to be disjoint from $L_{2}$.

\section{Claim 2.12 Either}

- there is a Möbius band in $X$ whose boundary is a meridian on $T_{1}$ or $T_{2}$; or

- there is an annulus in $X$ with a boundary component on each of $T_{1}$ and $T_{2}$ both of which are essential in $T_{1}, T_{2}$ and neither of which is isotopic to a meridian or to a component of $\partial Q$.

Proof By the above, we may assume there is a Möbius band $S$ in $M-\mathcal{N}\left(K \cup L_{1}\right)$ with meridional boundary in $T_{1}$. We assume there is no such $S$ disjoint from $L_{2}$ and take $S$ to intersect $L_{2}$ minimally. Let $S^{\prime}=S \cap X$. Isotope $\partial Q, \partial S^{\prime}$ to intersect minimally in $\partial X$. Then no arc of $Q \cap S^{\prime}$ is boundary parallel in $Q$ into $\partial Q \cap T_{2}$. Let $A$ be the punctured annulus coming from the boundary of a regular neighborhood of $S^{\prime}$ in $X$. Then no arc of $Q \cap A$ is boundary parallel in $Q$ into $\partial Q \cap T_{2}$ as there was no such for $Q \cap S^{\prime}$. Consider the graphs of intersection $G_{A}, G_{Q}^{\prime}$ coming from the arcs of $Q \cap A$ (as done for $G_{P}, G_{Q}$ ). Then $G_{Q}^{\prime}$ has no monogons based at the vertices corresponding to $T_{2}$. The parity rule shows that $G_{A}$ has no monogons. We now apply the argument above to $G_{A}, G_{Q}^{\prime}$ (in place of $G_{P}, G_{Q}$ ) to find a Möbius band, disk, or annulus in $X$.

To fit that argument (despite the slight awkwardness of indices), set

$$
V_{1}=\left|\partial Q \cap T_{2}\right|, \quad V_{2}=\left|\partial Q \cap T_{1}\right|, \quad m_{1}=\left|\partial A \cap T_{2}\right| \geq 2, \quad m_{2}=\left|A \cap T_{1}\right|=2 .
$$


Then $m_{1} \geq m_{2}$ and there are no monogons of $G_{Q}^{\prime}$ at any vertex of $V_{1}$. This corresponds to the situation in the above argument coming from conclusion (B) of Lemma 2.7 (with $A$ taking the role of $P$ ). Each vertex of $V_{1}$ in $G_{Q}^{\prime}$ has valence $m_{1} \Delta_{2}$. Let $G_{Q}^{\prime}\left(V_{1}\right)$ be the subgraph of $G_{Q}^{\prime}$ consisting of all edges of $G_{Q}^{\prime}$ that are incident to $V_{1}$ and all the vertices of $G_{Q}^{\prime}$ to which these edges are incident. Again $G_{Q}^{\prime}\left(V_{1}\right)$ is a graph in the surface gotten by attaching disks to $Q$ along those components of $\partial Q$ corresponding to vertices of $G_{Q}^{\prime}\left(V_{1}\right)$. Let $\widetilde{G}_{Q}^{\prime}\left(V_{1}\right)$ be the reduced graph obtained from $G_{Q}^{\prime}\left(V_{1}\right)$ by amalgamating parallel edges. By Claim 2.9, there must be at least $m_{1} \Delta_{2} / \max (-6 \chi(Q), 1)$ mutually parallel edges of $G_{Q}^{\prime}\left(V_{1}\right)$. Let $\mathcal{E}$ be one of these sets of edges. Since $A$ is disjoint from $K$, the edges in $\mathcal{E}$ either (a) join two vertices of $V_{1}$ (perhaps the same vertex) or (b) join a vertex of $V_{1}$ to a vertex of $V_{2}$. Let $G_{A}(\mathcal{E})$ be the subgraph of $G_{A}$ consisting of the edges in $\mathcal{E}$ and the vertices to which these edges are incident. For case (a), these vertices are all the $m_{1}$ vertices corresponding to $A \cap T_{2}\left(\Delta_{2}>\max (-6 \chi(Q), 1)\right)$. In this case we think of $G_{A}(\mathcal{E})$ as a graph in the annulus $\hat{A}$ gotten by abstractly capping off the components of $A \cap T_{2}$ with disks (ie $V_{2}$ corresponds to the boundary of $\left.\hat{A}\right)$. For case (b), since $\Delta_{2}>\max (-6 \chi(Q), 1)$ and $m_{1} \geq m_{2}$, the vertices of $G_{A}(\mathcal{E})$ are all the $m_{1}$ vertices corresponding to $A \cap T_{2}$ with both vertices of $A \cap T_{1}$. In case (b), we consider $G_{A}(\mathcal{E})$ as a graph in the 2-sphere $\hat{A}$ we get by abstractly capping off all of the boundary of $A$ with disks. In both cases (a) and (b) the vertices of $G_{A}(\mathcal{E})$ have valence at least $\Delta_{2} / \max (-6 \chi(Q), 1)$. (Each label of $G_{Q}^{\prime}$ at the vertices of $V_{1}$ or $V_{2}$ appears at least this many times at the endpoints of $\mathcal{E}$. For (b) we use that $m_{1} \geq m_{2}$.)

$G_{A}(\mathcal{E})$ has no monogons since $G_{A}$ has none. Also each vertex of $G_{A}(\mathcal{E})$ has valence at least

$$
\min \left(\Delta_{1}, \Delta_{2}\right) / \max (-6 \chi(Q), 1)>6\left(2 \mathfrak{b}_{\widehat{F}}(K) \max \left(\Delta_{K}, 1\right)+2 g-1\right) \geq 6
$$

Therefore Claim 2.10 (with $G=G_{A}(\mathcal{E})$ and $\hat{A}$ playing the role of $P$ ) implies that $G_{A}(\mathcal{E})$ has parallel edges. Hence there is a pair of edges $e, e^{\prime} \in \mathcal{E}$ that bounds a disk $D_{Q}$ in $G_{Q}^{\prime}$ and a disk $D_{A}$ in $G_{A}(\mathcal{E})$. We may assume $D_{Q} \cap D_{A}=e \cup e^{\prime}$.

Then, as above, we have two possibilities. In case (a), $D_{Q} \cup D_{A}$ is a Möbius band in $X$ with boundary a meridian on $T_{2}$ [6, Lemma 2.1]. In case (b), $D_{Q} \cup D_{A}$ is an annulus in $X$ with a boundary component on each of $T_{1}$ and $T_{2}$ both of which are essential in $T_{1}, T_{2}$ and neither of which is isotopic to a component of $\partial Q$ or $\partial A$. As $\partial A$ is meridional on each of $T_{1}$ and $T_{2}$, this completes the proof of the claim.

Claim 2.12 finishes the proof of Lemma 2.8. 


\subsection{Proof of main theorems}

In this section we prove Theorem 1.3, Corollary 1.1 and Corollary 1.4.

Theorem 1.3 Let $M$ be a compact, orientable 3-manifold with (possibly empty) boundary and $K \cup L_{1} \cup L_{2}$ a link in $M$. Let $\widehat{R}$ be an annulus in $M$ with $\partial \widehat{R}=L_{1} \cup L_{2}$. Assume $(\hat{R}, K)$ in $M$ is caught by the surface $Q$ in $X=M-\mathcal{N}\left(K \cup L_{1} \cup L_{2}\right)$. Let $T_{K}, T_{1}, T_{2}$ be the components of $\partial X$ corresponding to $K, L_{1}, L_{2}$ respectively. Fixing an orientation on $M$, let $\mu_{i}$ be a meridian of $L_{i}$ on $T_{i}$ and $\lambda_{i}$ be a framing curve coming from $\hat{R}$. Express the first homology class of a component of $\partial Q$ on $T_{i}$ as $p_{i}\left[\mu_{i}\right]+q_{i}\left[\lambda_{i}\right]$. Let $\Delta_{K}$ be the distance on $T_{K}$ between a component of $\partial Q$ and a meridian of $K$ (setting $\Delta_{K}=0$ when $Q$ is disjoint from $K$ ). Let $K^{n}$ be $K$ twisted $n$ times along $\widehat{R}$ (Definition 1.2).

Let $H_{1} \cup_{\widehat{F}} H_{2}$ be a genus- $g$ Heegaard splitting of $M$.

Then one of the following holds:

(1) $\hat{R}$ can be isotoped to lie in $\widehat{F}$.

(2) There is an essential annulus $A$ properly embedded in $X$ with a boundary component in each of $T_{1}$ and $T_{2}$. Furthermore, the slope of $\partial A$ on each $T_{i}$ is neither that of the meridian of $\mathcal{N}\left(L_{i}\right)$ nor that of $\partial Q$.

(3) For each $n$,

$$
\mathfrak{b}_{\widehat{F}}\left(K^{n}\right) \geq \frac{\min \left(\left|q_{1}+n p_{1}\right|,\left|q_{2}-n p_{2}\right|\right) / \max (-36 \chi(Q), 6)-2 g+1}{2 \max \left(\Delta_{K}, 1\right)}
$$

Proof Let $H_{1} \cup{ }_{\hat{F}} H_{2}$ be the given genus- $g$ Heegaard splitting of $M$. Let $K, K^{n}, L_{1}$, $L_{2}, \hat{R}, X$, and $Q$ be as stated. Let $R$ be the annulus $\hat{R} \cap\left(M-\mathcal{N}\left(L_{1} \cup L_{2}\right)\right)$. If $R$ is compressible in $M-\mathcal{N}\left(L_{1} \cup L_{2}\right)$, then $\widehat{R}$ can be isotoped onto $\widehat{F}$. We hereafter assume that $R$ is incompressible.

Dehn twists along the annulus $R$ provide homeomorphisms of $M-\mathcal{N}\left(L_{1} \cup L_{2}\right)$ in which the meridians of $L_{1}$ and $L_{2}$ are spun with opposite handedness around $\partial \hat{R}$. In particular, let $h_{n}: M-\mathcal{N}\left(L_{1} \cup L_{2}\right) \rightarrow M-\mathcal{N}\left(L_{1} \cup L_{2}\right)$ be the homeomorphism of Definition 1.2 obtained by twisting $n$ times along $R$. Define $\mathcal{L}^{n}$ to be the link $K^{n} \cup L_{1} \cup L_{2}$ and let $X_{n}$ be its exterior in $M$. Then $h_{n}$ induces a homeomorphism $h_{n}^{\prime}: X \rightarrow X_{n}$. Define $Q_{n}=h_{n}^{\prime}(Q)$.

Use the meridian, longitude coordinates to express the first homology class of a component of $\partial Q$ on $\partial \mathcal{N}\left(L_{i}\right)$ as $p_{i}\left[\mu_{i}\right]+q_{i}\left[\lambda_{i}\right]$. As $Q$ catches $(\hat{R}, K), p_{i} \neq 0$. With these same coordinates, the first homology class of a component of $\partial Q_{n}$ on $\partial \mathcal{N}\left(L_{i}\right)$ 
is $p_{i}\left[\mu_{i}\right]+\left(q_{i}+(-1)^{i+1} n p_{i}\right)\left[\lambda_{i}\right]$. In particular, the distance, $\Delta_{i}^{n}$, between a component of $\partial Q_{n}$ and the meridian $\mu_{i}$ on $\partial \mathcal{N}\left(L_{i}\right)$ is $\left|q_{i}+(-1)^{i+1} n p_{i}\right|$. Furthermore, the components of $\partial Q_{n}$ are coherently oriented on $\partial \mathcal{N}\left(L_{i}\right)$ since those of $Q$ are. In other words, $Q_{n}$ catches the pair $\left(\hat{R}, K^{n}\right)$ in $M$.

Lemma 2.13 If $L_{1} \cup L_{2}$ can be isotoped so that $L_{1}$ and $L_{2}$ lie on disjoint copies of $\widehat{F}$ of $M$ then $\hat{R}$ can be isotoped to lie in $\widehat{F}$.

Proof Isotope $L_{1}, L_{2}$ to lie in $\widehat{F}_{1}, \widehat{F}_{2}$, disjoint copies of $\hat{F}$. We may take $\hat{F}$ to lie between them. Isotope $\hat{R}$ so that it intersects $\widehat{F}$ transversely. Then some curve $c$ of $\widehat{R} \cap \widehat{F}$ will be a core curve of $\widehat{R}$. Hence $\widehat{R}$ can be isotoped to a neighborhood of $c$ and then into $\hat{F}$.

Thus we assume $L_{1} \cup L_{2}$ cannot be isotoped so that $L_{1}, L_{2}$ lie on disjoint copies of $\widehat{F}$. We apply Lemma 2.8 to $K^{n}, L_{1}, L_{2}, Q^{n}$. Note that conclusion (a) cannot hold because of the annulus $R$ between in $M-\mathcal{N}\left(L_{1} \cup L_{2}\right.$ ) (see eg Lemma 2.14 below). If conclusion (b) holds, then the annulus in $X$ must be essential in $X$ by the incompressibility of $R$ in $M-\mathcal{N}\left(L_{1} \cup L_{2}\right)$ (a compressing disk for the annulus in $X$ would give rise to one for $R$ ). Thus conclusion (b) gives conclusion (2), and we may assume (b) does not hold. Thus we must conclude that

$$
\begin{aligned}
\min \left(\left|q_{1}+n p_{1}\right|,\left|q_{2}-n p_{2}\right|\right) & =\min \left(\Delta_{1}^{n}, \Delta_{2}^{n}\right) \\
& \leq \max \left(-36 \chi\left(Q^{n}\right), 6\right)\left(2 \mathfrak{b}_{\hat{F}}\left(K^{n}\right) \max \left(\Delta_{K^{n}}, 1\right)+2 g-1\right) .
\end{aligned}
$$

As $\Delta_{K^{n}}=\Delta_{K}$ and $\chi\left(Q^{n}\right)=\chi(Q)$ we may rewrite this as

$$
\mathfrak{b}_{\widehat{F}}\left(K^{n}\right) \geq \frac{\min \left(\left|q_{1}+n p_{1}\right|,\left|q_{2}-n p_{2}\right|\right) / \max (-36 \chi(Q), 6)-2 g+1}{2 \max \left(\Delta_{K}, 1\right)}
$$

as desired.

This completes the proof of Theorem 1.3.

We need the following for the proof of Corollary 1.1.

Lemma 2.14 Let $N$ be an orientable 3-manifold with toral boundary components $T_{1}, T_{2}$ ( $\partial N$ may contain other components). Let $A$ be an incompressible annulus in $N$ with a boundary component on each of $T_{1}$ and $T_{2}$. Let $B$ be a $\partial$-incompressible annulus or a Möbius band in $N$, in either case with essential boundary on $T_{1} \cup T_{2}$. Then either:

- Each component of $\partial B$ must be isotopic on $T_{1} \cup T_{2}$ to one of $\partial A$.

- $\partial B$ has a component on each of $T_{1}$ and $T_{2}$ and $N$ is either $T^{2} \times[0,1]$ or has $T^{2} \times[0,1]$ as a connected summand, where $T^{2} \times\{0,1\}$ is $T_{1} \cup T_{2}$. 
Proof Since $A$ is incompressible there is no essential disk in $N$ with boundary on $T_{1} \cup T_{2}$.

First, assume $\partial B$ lies on $T_{i}$ and no component is isotopic to $\partial A \cap T_{i}$. Isotope $\partial B$ to intersect $\partial A$ minimally on $T_{i}$. After possibly surgering $B$ along trivial simple closed curves of intersection with $A$, a disk in $A$ bounded by an outermost $\operatorname{arc}$ of $A \cap B$ gives a $\partial$-compressing disk for $B$. Then $B$ must be a Möbius band and $\partial-$ compressing $B$ gives an essential disk in $N$ with boundary on $T_{1} \cup T_{2}$, a contradiction.

So we assume that $\partial B$ has one component on $T_{1}$ and another on $T_{2}$. Note that $B$ must be incompressible in $N$ (else there is an essential disk at $T_{1}$ or $T_{2}$ in $N$ ). Isotope $\partial B, \partial A$ on $T_{1} \cup T_{2}$ to intersect minimally. Surger $A, B$ so that no closed curves of intersection are trivial in either $A$ or $B$. By orientability (the parity rule), each arc of $A \cap B$ must connect different components of $\partial A$ and different components of $\partial B$. Thus $A \cap B$ is a collection of parallel spanning $\operatorname{arcs}$ in $A$ and in $B$. Take a pair that cobound a disk $D_{1}$ of $A-B$. These $\operatorname{arcs}$ in $B$ then cobound a disk $D_{2}$ in $B$. Then $D_{1} \cup D_{2}$ gives an annulus $C$ between $T_{1} \cup T_{2}$ such that $\partial C$ can be isotoped to intersect $\partial B$ once on each of $T_{1}$ and $T_{2}$. Indeed, we may isotope $C$ so that it intersects $B$ in a single arc. Then $\mathcal{N}\left(C \cup B \cup T_{1} \cup T_{2}\right)$ has a 2 -sphere boundary component that displays $N$ as a connected sum with $T_{1} \times[0,1]$ as claimed.

In terms of genus- $g$ bridge numbers, Theorem 1.3 has a partial converse. (In the statement below we have added some explanatory notation to the version of Corollary 1.1 stated in the introduction.)

Corollary 1.1 Let $M$ be a compact, orientable 3-manifold with (possibly empty) boundary and $K \cup L_{1} \cup L_{2}$ be a link in $M$. Let $\widehat{R}$ be an annulus in $M$ with $\partial \widehat{R}=L_{1} \cup L_{2}$, and let $R$ be the annulus $\widehat{R} \cap\left(M-\mathcal{N}\left(L_{1} \cup L_{2}\right)\right)$ properly embedded in $M-\mathcal{N}\left(L_{1} \cup L_{2}\right)$. Assume $(\widehat{R}, K)$ in $M$ is caught. Let $X=M-\mathcal{N}\left(K \cup L_{1} \cup L_{2}\right)$ and $T_{K}, T_{1}, T_{2}$ be the components of $\partial X$ corresponding to $K, L_{1}, L_{2}$ respectively. Fixing an orientation on $M$, let $K^{n}$ be $K$ twisted $n$ times along $\hat{R}$.

Assume $M$ has a genus- $g$ Heegaard splitting.

Then one of the following holds:

(1) There is a genus- $g$ Heegaard surface of $M$ containing $\hat{R}$.

(2) There is an essential annulus $A$ in $X$ with one component of $\partial A$ on $T_{1}$ and the other on $T_{2}$ such that $\partial A$ and $\partial R$ are isotopic on $T_{1} \cup T_{2}$.

$\mathfrak{b}_{g}\left(K^{n}\right) \rightarrow \infty$ as $n \rightarrow \infty$.

Furthermore, if either (1) or (2) holds, then $\left\{\mathfrak{b}_{g}\left(K^{n}\right)\right\}$ is a finite set. 
Proof Assume that conclusion (3) above does not hold. Then there is genus- $g$ Heegaard surface $\widehat{F}$ of $M$ that fails inequality (3) of Theorem 1.3. Then Theorem 1.3 proves the corollary unless there is an essential annulus $A$ in $X$ with one component of $\partial A$ on $T_{1}$ and the other on $T_{2}$. We may also assume that $R$ is essential in $M-\mathcal{N}\left(L_{1} \cup L_{2}\right)$, as otherwise conclusion (1) of the corollary will hold. We show that $\partial A$ must have the same slopes as $\partial R$ on $T_{1}$ and $T_{2}$, giving conclusion (2). Assume not. Then $\partial A$ must have different slopes on both $T_{1}$ and $T_{2}$ from $\partial R$. Thus we may apply Theorem 1.3 using $A$ as the catching surface for $\hat{R}$. Again, this proves the corollary unless there is another essential annulus $A^{\prime}$ in $X$ whose boundary has different slopes on $T_{1}$ and $T_{2}$ from $\partial A$. Applying Lemma 2.14 to $X$ shows that $X$ has $T^{2} \times[0,1]$ as a connected summand, where $T^{2} \times\{0,1\}$ is $T_{1} \cup T_{2}$. Thus there is a 2-sphere in $X$ separating $T_{K}$ from $T_{1} \cup T_{2}$ and we may surger $R \cap X$ along this 2-sphere to obtained an essential annulus in $X$ with the same boundary as $R$, as desired.

We must show that if (1) or (2) hold, then $\left\{\mathfrak{b}_{g}\left(K^{n}\right)\right\}$ is a finite set. Assume (1) holds, and let $S$ be a genus- $g$ Heegaard surface containing $\widehat{R}$. Now isotope $K$, keeping $\widehat{R}$ fixed, so that it is bridge with respect to $S$. Then $\left\{\mathfrak{b}_{g}\left(K^{n}\right)\right\}$ will be finite since each element is bounded above by the bridge number of this representative of $K$.

Assume that (2) holds. Let $M^{n}=M\left(-\frac{1}{n}, \frac{1}{n}\right)$ be the result of $\left(-\frac{1}{n}, \frac{1}{n}\right)-$ Dehn surgery on $L_{1}, L_{2}$ in $M$ using the framings given by $R$. As in Definition 1.2 , there is a homeomorphism $h_{n}$ of $M-\mathcal{N}\left(L_{1} \cup L_{2}\right)$ to itself that induces $h_{n}^{\prime}: M^{n} \rightarrow M$. Furthermore, $h_{n}^{\prime}$ identifies the pair $\left(M^{n}, K\right)$ with the pair $\left(M, K^{n}\right)$. In the same way, twisting along $A$ induces a homeomorphism $f_{n}^{\prime}: M^{n} \rightarrow M$ identifying the pair $\left(M^{n}, K\right)$ with $(M, K)$. Thus $\mathfrak{b}_{g}\left(K^{n}\right)=\mathfrak{b}_{g}(K)$ for each $n$.

Remark 2.15 Assume that conclusions (1) and (2) of Corollary 1.1 do not hold. The proof of Corollary 1.1 shows that either:

(A) For each $n$,

$$
\mathfrak{b}_{g}\left(K^{n}\right) \geq \frac{\min \left(\left|q_{1}+n p_{1}\right|,\left|q_{2}-n p_{2}\right|\right) / \max (-36 \chi(Q), 6)-2 g+1}{2 \max \left(\Delta_{K}, 1\right)},
$$

where $\left(p_{i}, q_{i}\right)$ are the coordinates of $\partial Q$ on $T_{i}$ (framed by $R$ as above) and $\Delta_{K}$ is the distance on $T_{K}$ between a component of $\partial Q$ and a meridian of $K$ (setting $\Delta_{K}=0$ when $Q$ is disjoint from $K$ ).

(B) There is an annular catching surface $Q^{\prime}$ for $\hat{R}$ in $M$. Let $\left(r_{i}, s_{i}\right)$ be the coordinates of $\partial Q^{\prime}$ on $T_{i}$ (framed by $R$ ). Then, for each $n$,

$$
\mathfrak{b}_{g}\left(K^{n}\right) \geq \frac{1}{12} \min \left(\left|s_{1}+n r_{1}\right|,\left|s_{2}-n r_{2}\right|\right)-g+\frac{1}{2} .
$$

We finish with the proof of the following. 
Corollary 1.4 Assume that $M$ is closed and orientable and let $K \cup L_{1} \cup L_{2}$ be a link in $M$. Let $\hat{R}$ be an annulus in $M$ with $\partial \widehat{R}=L_{1} \cup L_{2}$, and let $R$ be the annulus $\widehat{R} \cap\left(M-\mathcal{N}\left(L_{1} \cup L_{2}\right)\right)$ properly embedded in $M-\mathcal{N}\left(L_{1} \cup L_{2}\right)$. Assume that $(\hat{R}, K)$ in $M$ is caught. Assume there is no properly embedded, essential annulus $A$ in $X=M-\mathcal{N}\left(K \cup L_{1} \cup L_{2}\right)$ such that $\partial A \cap\left(T_{1} \cup T_{2}\right)$ is isotopic to $\partial R \cap\left(T_{1} \cup T_{2}\right)$ on $T_{1} \cup T_{2}$. Fixing an orientation on $M$, let $K^{n}$ be $K$ twisted $n$ times along $\hat{R}$. If $M=S^{3}$ and $L_{1} \cup L_{2}$ is not the trivial link, then $\mathfrak{b}_{0}\left(K^{n}\right) \rightarrow \infty$ as $n \rightarrow \infty$.

If $M$ is a lens space and $L_{1} \cup L_{2}$ is not a lens space torus link, then $\mathfrak{b}_{1}\left(K^{n}\right) \rightarrow \infty$ as $n \rightarrow \infty$.

If $M$ has Heegaard genus at most 2, then either $\mathfrak{b}_{2}\left(K^{n}\right) \rightarrow \infty$ as $n \rightarrow \infty$ or one of the following holds:

(a) $L_{1}$ has tunnel number one in $M$ (or bounds a disk in $M$ ).

(b) $L_{1}$ is a cable of a tunnel number one knot in $M$ where the slope of the cabling annulus is that of $\partial R$.

(c) 0-surgery (as framed by $R$ ) on $L_{1}$ contains an essential torus.

As $L_{1}$ and $L_{2}$ are isotopic in $M$, if any of (a)-(c) holds for $L_{1}$, then it also holds for $L_{2}$.

Proof Under the hypotheses given, Corollary 1.1 implies that if $\mathfrak{b}_{g}\left(K^{n}\right)$ does not tend to infinity with $n$ then $\hat{R}$ lies on a genus- $g$ Heegaard splitting $H_{1} \cup_{\widehat{F}} H_{2}$ of $M$. The conclusions for $g=0$ and $g=1$ are then immediate. So assume $g=2$.

If $F=\widehat{F}-\mathcal{N}\left(L_{1}\right)$ is compressible in the complement of $L_{1}$, then such a compression shows that $L_{1}$ is a cable of a core of either $H_{1}$ or $H_{2}$. In this case either $L_{1}$ has tunnel number one or is the cable of a tunnel number one knot. If on the other hand $F$ is incompressible then the handle addition lemma [4, Lemma 2.1.1] implies that surgery on $L_{1}$ along the slope induced by $F$ is toroidal.

\section{Application to Teragaito's example and some generaliza- tions}

Osoinach describes a construction producing infinitely many distinct knots in $S^{3}$ (or some other manifold) for which the same integral surgery on each knot yields the same new manifold $M$ [14]. Dually, this may be viewed as infinitely many distinct knots in a manifold $M$ (that is, no homeomorphism of $M$ takes one knot to the other) for which the same integral surgery yields $S^{3}$. Teragaito gives a specific example 

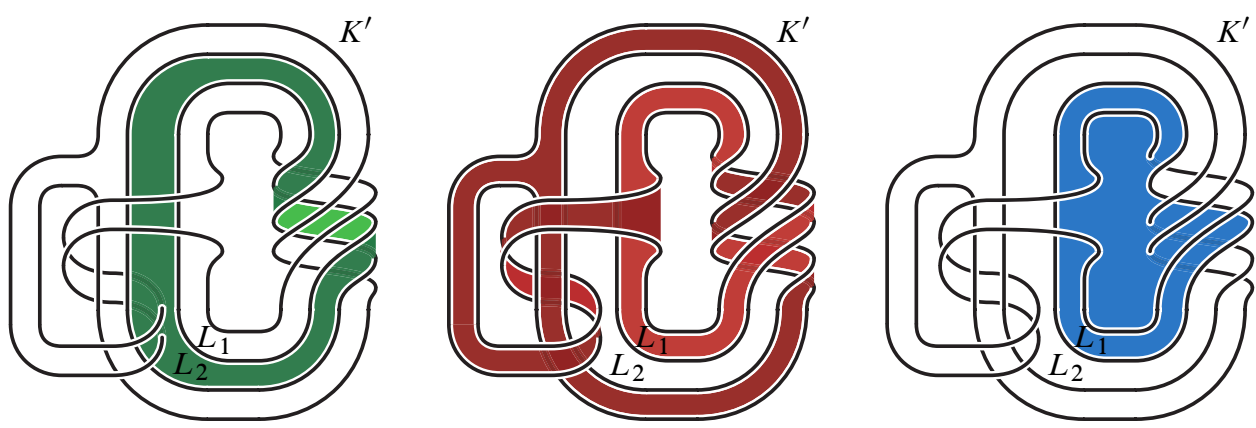

Figure 3: The link $\mathcal{L}^{\prime}=K^{\prime} \cup L_{1} \cup L_{2}$ shown with the green annulus $A$, the red pair of pants $R$, and the blue disk $\hat{Q}$.

of this construction in which the manifold $M$ is a small Seifert fiber space [17]. We produce a two-parameter generalization of Teragaito's examples in which the resulting manifolds $M$ have Heegaard genus 2 and are typically hyperbolic. We apply Corollary 1.4 to show that Teragaito's family of knots and our generalizations (for large parameter values) have genus- 2 bridge numbers in $M$ that tend to infinity. Let us first review Teragaito's example.

Teragaito describes a 3-component link $\mathcal{L}^{\prime}=K^{\prime} \cup L_{1} \cup L_{2}$ in $S^{3}$ where $L_{1} \cup L_{2}$ is the boundary of an annulus $A$ and there is a pair of pants $R$ (that intersects the interior of $A$ ) expressing $K^{\prime}$ as a banding of $L_{1} \cup L_{2}$ and meeting $L_{1} \cup L_{2}$ with the same framing as $A$, as shown in Figure 3. Frame the components of the link $\mathcal{L}^{\prime}$ with $R$. Then, as Teragaito shows, 0 -surgery on $K^{\prime}$ (that is, a +4 -surgery with respect to the Seifert framing) produces a small Seifert fiber space $M$ containing the knot $K$ dual to the surgery and an annulus $\hat{R}$ with boundary $L_{1} \cup L_{2}$. The annulus $\hat{R}$ is obtained after surgery by capping off the $K^{\prime}$ component of $\partial R$ with a disk. Indeed, the interior of $\widehat{R}$ is pierced once by $K$ in $M$.

We generalize Teragaito's example by inserting extra twists in two regions. Figure 4 is the same as Figure 3 except that two (unlinked) unknots $J_{0}$ and $J_{1}$ have been added and the full twist on the right-hand side of the link $\mathcal{L}^{\prime}$ has been undone (which may be restored by a -1 -surgery along $J_{1}$ ). Produce the link

$$
\mathcal{L}_{j_{0}, j_{1}}^{\prime}=K_{j_{0}, j_{1}}^{\prime} \cup\left(L_{1}\right)_{j_{0}, j_{1}} \cup\left(L_{2}\right)_{j_{0}, j_{1}} \quad \text { in } S_{j_{0}, j_{1}}^{3} \cong S^{3}
$$

by performing $-1 / j_{0}$-surgery on $J_{0}$ and $-1 / j_{1}$-surgery on $J_{1}$. The link $\mathcal{L}_{0,1}^{\prime}=\mathcal{L}^{\prime}$ is the one used by Teragaito. As one may conclude from Figure 4, $\left(L_{1}\right)_{j_{0}, j_{1}} \cup\left(L_{2}\right)_{j_{0}, j_{1}}$ cobound a green annulus $A_{j_{0}, j_{1}}$ and there is a red pair of pants $R_{j_{0}, j_{1}}$ (intersecting the interior of $A_{j_{0}, j_{1}}$ ) expressing $K_{j_{0}, j_{1}}^{\prime}$ as a banding of $\left(L_{1}\right)_{j_{0}, j_{1}} \cup\left(L_{2}\right)_{j_{0}, j_{1}}$ and 
meeting $\left(L_{1}\right)_{j_{0}, j_{1}} \cup\left(L_{2}\right)_{j_{0}, j_{1}}$ in the same framing as $A_{j_{0}, j_{1}}$. The component $J_{0}$ links the banding so that $-1 / j_{0}$-surgery on $J_{0}$ inserts $j_{0}$ full twists into the band.

Frame the components of the link $\mathcal{L}_{j_{0}, j_{1}}^{\prime}$ with $R_{j_{0}, j_{1}}$. (Observe that each of the link components of Figure 4 is an unknot and the framing induced by $R$ is the standard Seifert framing. Twisting along $J_{0}$ and $J_{1}$ will twist these framings.) Then 0 -surgery on $K_{j_{0}, j_{1}}^{\prime}$ produces a manifold $M_{j_{0}, j_{1}}$ containing the knot $K_{j_{0}, j_{1}}$ dual to the surgery and an annulus $\widehat{R}_{j_{0}, j_{1}} \subset M_{j_{0}, j_{1}}$ with boundary $\left(L_{1}\right)_{j_{0}, j_{1}} \cup\left(L_{2}\right)_{j_{0}, j_{1}}$. The annulus $\widehat{R}_{j_{0}, j_{1}}$ is obtained after surgery by capping off the $K_{j_{0}, j_{1}}^{\prime}$ component of $\partial R_{j_{0}, j_{1}}$; the interior of $\widehat{R}_{j_{0}, j_{1}}$ is pierced once by $K_{j_{0}, j_{1}}$ in $M_{j_{0}, j_{1}}$.

As $\left(L_{1}\right)_{j_{0}, j_{1}}$ is an unknot in $S^{3}$, it bounds a disk $\widehat{Q}_{j_{0}, j_{1}}$. This disk is punctured $2\left|j_{1}\right|$ times by $K_{j_{0}, j_{1}}^{\prime}$ and $\left|j_{1}\right|$ times by $\left(L_{2}\right)_{j_{0}, j_{1}}$. Let $X_{j_{0}, j_{1}}$ be the exterior of the link $\mathcal{L}_{j_{0}, j_{1}}^{\prime}$ in $S^{3}$. Let $Q_{j_{0}, j_{1}}=\widehat{Q}_{j_{0}, j_{1}} \cap X_{j_{0}, j_{1}}$ be this $3\left|j_{1}\right|$-punctured disk properly embedded in $X_{j_{0}, j_{1}}$, suggested in blue by the right-hand picture in Figure 4. The blue 3-punctured disk $Q_{0,1}$ is shown in Figure 3.

Let us now drop the subscripts $j_{0}, j_{1}$ from our notation except when needed. Thus, hereafter $K^{\prime}, L_{1}, L_{2}, A, R, M, X$ correspond to those with subscripts $j_{0}, j_{1}$.

$A$ is an annulus in $S^{3}$ with $\partial A=L_{1} \cup L_{2}$ and $\widehat{R}$ is an annulus in $M$ with $\partial \widehat{R}=L_{1} \cup L_{2}$. Twisting $K^{\prime}$ along $A$ produces the family of knots $\left\{K^{\prime n}\right\}$ in $S^{3}$, and twisting $K$ along $\hat{R}$ produces the family $\left\{K^{n}\right\}$ in $M$. Let $M_{n}$ (resp. $S_{n}^{3}$ ) be the manifold obtained from $M$ (resp. $S^{3}$ ) by $-1 / n$-surgery on $L_{1}$ and $1 / n$-surgery on $L_{2}$. In both $M_{n}$ and $S_{n}^{3}$ we continue to use the names $L_{1}$ and $L_{2}$ for the knots dual to these Dehn surgeries. As in Definition 1.2, there are homeomorphisms identifying the pair $\left(M_{n}, K \cup L_{1} \cup L_{2}\right)$ with $\left(M, K^{n} \cup L_{1} \cup L_{2}\right)$ and the pair $\left(S_{n}^{3}, K^{\prime} \cup L_{1} \cup L_{2}\right)$ with $\left(S^{3}, K^{\prime n} \cup L_{1} \cup L_{2}\right)$. Use the framing on $K^{\prime}$ (by $R$ ) and the identification $\left(S_{n}^{3}, K^{\prime}\right) \cong\left(S^{3}, K^{\prime n}\right)$ to assign a framing to $K^{\prime n}$. Then the knot dual to the 0 -surgery on $K^{\prime n}$ in $S^{3}$ is the knot dual to the 0 -surgery on $K^{\prime}$ in $S_{n}^{3}$, which by definition is $K$ in $M_{n}$. But this is identified with $K^{n}$ in $M$. That is, we see that $K^{n}$ is the dual knot to the 0-surgery on $K^{\prime n}$. Finally, observe that

$$
\begin{aligned}
X & =S^{3}-\mathcal{N}\left(K^{\prime} \cup L_{1} \cup L_{2}\right) \\
& \cong S^{3}-\mathcal{N}\left(K^{\prime n} \cup L_{1} \cup L_{2}\right) \\
& \cong M-\mathcal{N}\left(K^{n} \cup L_{1} \cup L_{2}\right) \\
& \cong M-\mathcal{N}\left(K \cup L_{1} \cup L_{2}\right)
\end{aligned}
$$

Theorem 1.5 Let $\left\{K^{\prime n}\right\}$ be the Teragaito family of knots in $S^{3}$. For each $n$, let $K^{n} \subset M$ be the +4 -surgery-dual to $K^{\prime n}$ with respect to the Seifert framing on $K^{\prime n}$. Then $\mathfrak{b}_{0}\left(K^{\prime n}\right) \rightarrow \infty$ and $\mathfrak{b}_{2}\left(K^{n}\right) \rightarrow \infty$ as $n \rightarrow \infty$. 

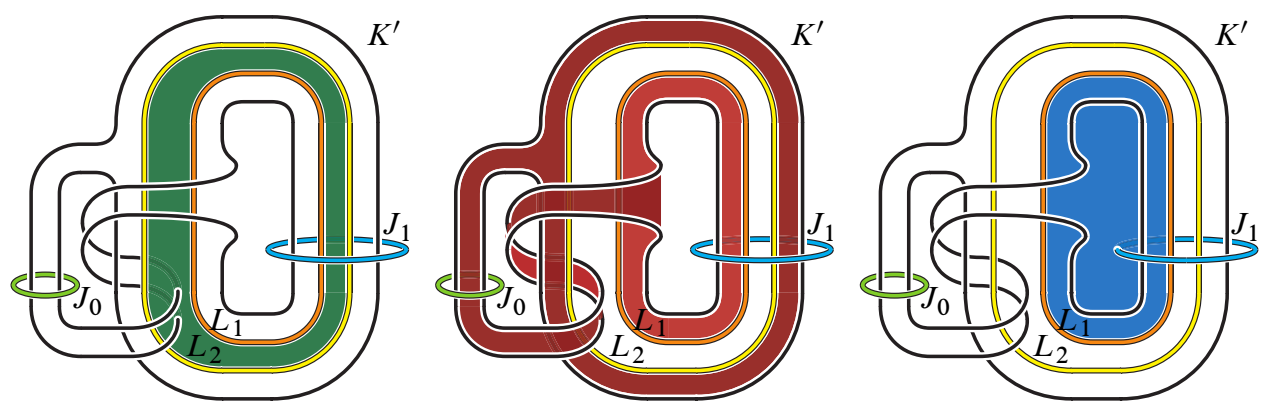

Figure 4: The link $\mathcal{L}^{\prime}=K^{\prime} \cup L_{1} \cup L_{2}$ shown with the green annulus $A$, the red pair of pants $R$, and the blue disk $\hat{Q}$.

Proof Recall that the Teragaito family is where $j_{0}=0, j_{1}=1$ and the +4 -surgery in the Seifert framing is our 0 -surgery when framed by $R$. The 3-punctured disk $Q$ in the exterior $X$ obtained from the disk $\hat{Q}$ has one component of its boundary on $\partial \mathcal{N}\left(L_{1}\right)$ and one component of its boundary on $\partial \mathcal{N}\left(L_{2}\right)$. As $j_{1} \neq 0$, these slopes both differ from the slopes of $\partial \hat{R}$, and so $Q$ catches $(\hat{R}, K)$ and $\left(A, K^{\prime}\right)$.

Teragaito shows that the link exterior $X$ of $K^{\prime} \cup L_{1} \cup L_{2}$ in $S^{3}$ (and of $K \cup L_{1} \cup L_{2}$ in $M$ ) is hyperbolic; hence in particular $X$ contains no essential annulus. We apply Corollary 1.4. As $L_{1} \cup L_{2}$ is not trivial in $S^{3}, \mathfrak{b}_{0}\left(K^{\prime n}\right) \rightarrow \infty$ as $n \rightarrow \infty$. By Lemma $3.4, \mathfrak{b}_{2}\left(K^{n}\right) \rightarrow \infty$ as $n \rightarrow \infty$.

The Teragaito family $\left\{K^{n}\right\}$ is thus a family of knots in the Seifert fiber space $M$ of unbounded bridge number each of which nevertheless admits an $S^{3}$ surgery. We show that the above generalization yields such families of knots (arbitrarily large genus- 2 bridge number where each knot admits an $S^{3}$ surgery) in manifolds $M$ which are hyperbolic.

Definition 3.1 SnapPy [3] shows that the manifold $W=S^{3}-\mathcal{N}\left(K^{\prime} \cup J_{0} \cup J_{1}\right)$ of Figure 4 is hyperbolic. It also verifies that $W_{0}$, the Dehn filling of $W$ along the slope of $\partial R$ (ie slope 0 ) on the component of $\partial W$ coming from $\partial \mathcal{N}\left(K^{\prime}\right)$, is hyperbolic. SnapPy also shows that $Y=S^{3}-\mathcal{N}\left(K^{\prime} \cup J_{0} \cup J_{1} \cup L_{2}\right)$ is hyperbolic. By Thurston's hyperbolic Dehn surgery theorem, there is a constant $v$, which we will take to be greater than 2 , such that the following hold as long as $\min \left\{\left|j_{0}\right|,\left|j_{1}\right|\right\} \geq v$ :

- $M$, which is the Dehn filling of $W_{0}$ along the slopes $-1 / j_{0}$ and $-1 / j_{1}$ on the components of $W_{0}$ coming from $\partial \mathcal{N}\left(J_{0}\right)$ and $\partial \mathcal{N}\left(J_{1}\right)$, respectively, is hyperbolic.

- $\quad Y_{j_{0}}$, the $-1 / j_{0}-$ Dehn filling of $Y$ along the component of $\partial Y$ coming from $\partial \mathcal{N}\left(J_{0}\right)$, is hyperbolic. 
Theorem 3.2 For $\left|j_{0}\right|,\left|j_{1}\right| \geq v, M$ has Heegaard genus 2 and $\mathfrak{b}_{2}\left(K^{n}\right) \rightarrow \infty$ as $n \rightarrow \infty$.

Proof This follows from Corollary 1.4, Lemma 3.3 and Lemma 3.4.

Lemma 3.3 Let $X=M-\mathcal{N}\left(K^{n} \cup L_{1} \cup L_{2}\right)$ and $T_{1}, T_{2}$ the components of $\partial X$ corresponding to $\partial \mathcal{N}\left(L_{1}\right), \partial \mathcal{N}\left(L_{2}\right)$. If $\left|j_{0}\right| \geq v, j_{1} \neq 0$, there is no essential annulus in $X$ with one boundary component on $T_{1}$ and the other on $T_{2}$.

Proof As $L_{1}$ is isotopic to a meridian of $J_{1}$ in Figure 4, we can write $X=Y_{j_{0}} \cup_{T}$ $C\left(\left|j_{1}\right|, r\right)$, where $Y_{j_{0}}$ is as in Definition 3.1, $C\left(\left|j_{1}\right|, r\right)$ is cable space (a Seifert fibered space over the annulus with one exceptional fiber) between $T$ and $T_{1}$, and $T$ corresponds to $\partial \mathcal{N}\left(J_{1}\right)$ in $\partial Y_{j_{0}}$. An essential annulus in $X$ with one boundary component on $T_{1}$ and the other on $T_{2}$ would give rise to an essential annulus in $Y_{j_{0}}$ with boundary on $T$ and $T_{2}$ ( $T$ is incompressible in $Y_{j_{0}}$ ). But this contradicts the hyperbolicity of $Y_{j_{0}}$.

In support of the above theorems we proceed to show:

Lemma 3.4 For any $j_{0}, j_{1}, M$ has Heegaard genus 2. Furthermore, if either (a) $j_{1}= \pm 1$ and $\left|j_{0}\right| \neq 1,2$, or (b) $\left|j_{0}\right|,\left|j_{1}\right| \geq v \geq 3$, then for each $i \in\{1,2\}$ the link component $L_{i} \subset M$

- has tunnel number greater than 1 ,

- is not a cable of a tunnel number one knot where the slope of the cabling annulus is that of $\partial \hat{R}$, and

- has an atoroidal Dehn surgery along the slope $\partial \hat{R}$.

Proof We assume that $i=1$. Then the annulus $\hat{R}$ shows that the same statements hold for $L_{2}$.

(1) $M$ has Heegaard genus 2 To start, Figure 5 shows an isotopy of $K^{\prime} \cup L_{1} \cup$ $L_{2} \cup J_{0} \cup J_{1}$ into a simplified configuration. After dropping $L_{2}$, performing a further isotopy makes the remaining link $K^{\prime} \cup L_{1} \cup J_{0} \cup J_{1}$ strongly invertible, as shown in the first picture of Figure 6. The second picture continues the isotopy and exhibits the fixed set of this strong inversion. Each component of the link is an unknot bounding a disk that is also invariant under the involution. The framings of these disks agree with 

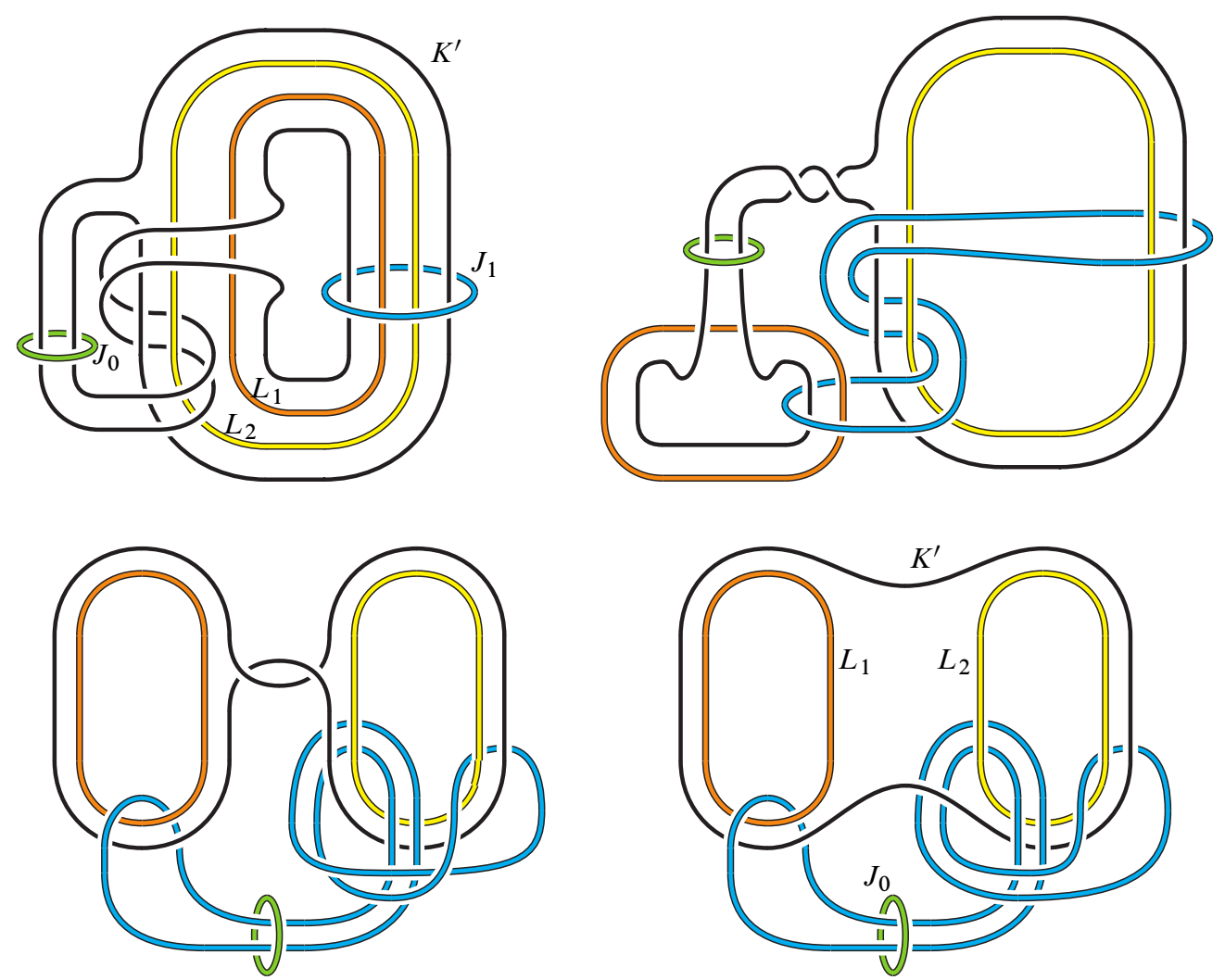

Figure 5

their page framings (ie blackboard framings). The third picture shows the quotient of the involution. Each link component except $J_{1}$ projects to an arc with the page framing. The fourth picture shows an isotopy of the arc $J_{1}$ and the fixed set that restores its framing to the page framing.

The second row of Figure 6 begins with a banding along (the arc corresponding to) $K^{\prime}$ using its page framing. By the Montesinos trick, this is equivalent to doing 0-surgery on $K^{\prime}$. The remaining sequence of pictures of Figure 6 up to the penultimate one exhibit isotopies of the fixed set and the arcs corresponding to $L_{1} \cup J_{0} \cup J_{1}$. Throughout these isotopies the page framings of the arcs are unaltered.

The final picture of Figure 6 replaces the horizontal arcs $J_{0}$ and $J_{1}$ with rectangles indicating vertical runs of $\left|j_{0}\right|$ and $\left|j_{1}\right|$ half-twists. The signs of $j_{0}$ and $j_{1}$ dictate the handedness of the twists as illustrated in Figure 7 . These replacements correspond to performing $-1 / j_{0}$ and $-1 / j_{1}$ surgeries on $J_{0}$ and $J_{1}$ in the double branched cover. 

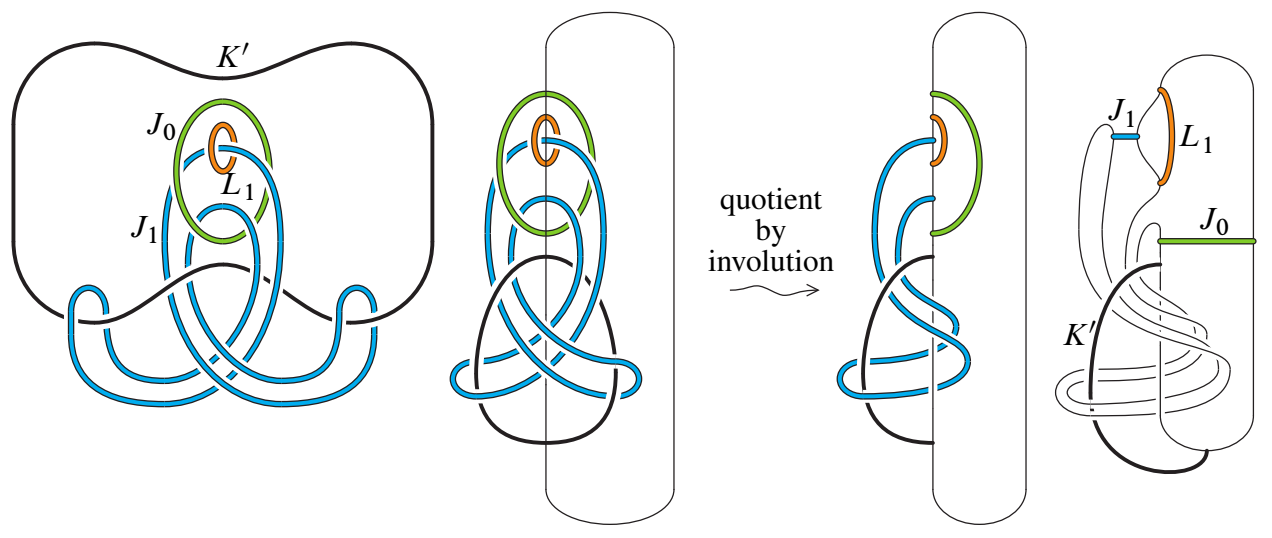

0 -surgery on $K^{\prime}$
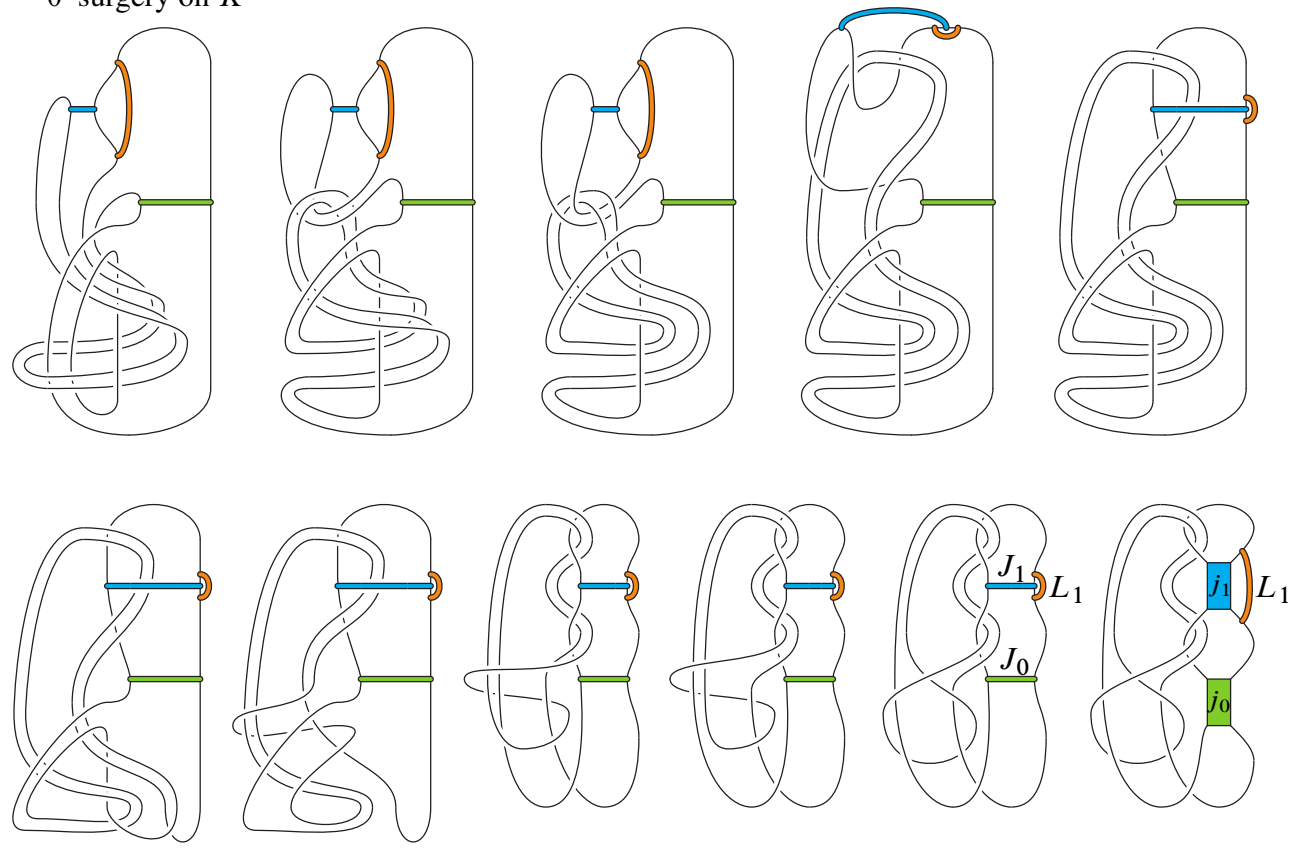

Figure 6

The double branched cover of the resulting link $\ell$ is the manifold $M=M_{j_{0}, j_{1}}$. Since the link $\ell$ is 3 -bridge, $M$ is a manifold of Heegaard genus at most 2.

(2) $L_{1}$ does not have tunnel number one The arc $L_{1}$ in the final picture of Figure 6 lifts to the knot $L_{1}$ in $M$. The exterior of a small ball neighborhood of the $\operatorname{arc} L_{1}$ is a ball that intersects the fixed set of the quotient in a tangle $\omega$. The double cover of this outside ball branched over $\omega$ is $M-\mathcal{N}\left(L_{1}\right)$. 

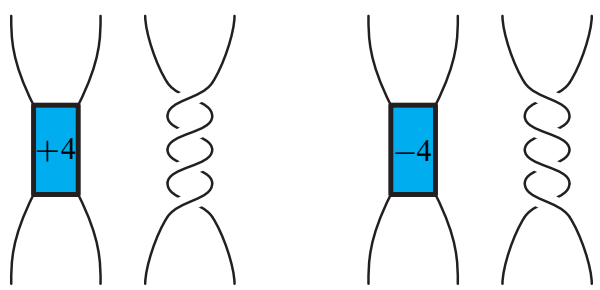

Figure 7

When $\left|j_{1}\right|=1$, the tangle $\omega$ is isotopic to the tangle $\tau$ of Claim 3.6, which shows that its double branched cover does not have tunnel number one.

So assume $\left|j_{1}\right|>1$ and that $M-\mathcal{N}\left(L_{1}\right)$ has tunnel number one. The double branched cover of the link we get by adding a rational tangle to $\omega$ is a Dehn filling of $M-\mathcal{N}\left(L_{1}\right)$. Any such Dehn filling must have Heegaard genus at most two. Consider the link $l$ gotten by adding $n>2$ vertical twists to $\omega$. The resulting link is the union of $\tau$ (Figure 8 ) and $\mu$, where $\mu$ is a non-rational tangle whose double branched cover is a Seifert fiber space $S$ over the disk with exceptional fibers of order $\left|j_{1}\right|, n$. The Seifert fiber of $S$ is unique up to isotopy along $\partial S$. By Claim 3.6, the Heegaard genus- 2 manifold $M_{l}$ that is the double branched cover of $l$ is the union along an incompressible torus $T$ of $N$ (the double branched cover of $\tau$ ) and $S$. As $N$ and $S$ are irreducible, so is $M_{l}$.

Convention Below we will be considering the links in $S^{3}$ gotten by filling the boundary sphere of tangles $\tau, \tau_{1}$ in Figures 8,10 with rational tangles. Rational tangles are determined by the slopes of their arcs on the bounding sphere with four marked points. For an integer $n$, our convention will be that the rational tangles (or the corresponding slopes on the sphere) are labeled: $n / 1$ for two horizontal arcs with $n$ right-handed twists, $1 / n$ for two vertical arcs with $n$ left-handed twists. On the level of the double branched covers, a slope on the tangle sphere determines a slope on the bounding torus above, and a tangle filling results in a Dehn filling along that slope.

First we show that $M_{l}$ cannot be a Seifert fiber space. Otherwise the separating torus $T$ would have to be vertical in that Seifert fiber space and $N$ would admit a Seifert fibration whose fiber agrees with the Seifert fiber of $S$. But Dehn filling $N$ along this fiber gives $S^{2} \times S^{1}$ (adding the $\frac{1}{0}$-tangle to $\tau$ gives the unlink). This means that $N$ is the circle bundle over the Möbius band. Thinking of $N$ as a Seifert fiber space over the disk with two exceptional fibers, each of order 2, we see that no Dehn filling of $N$ contains a separating essential torus. But filling $\tau$ with the $\frac{0}{1}-$ tangle gives a link that is a union of two tangles $\tau_{1}, \tau_{2}$ whose double branched cover contains a 
separating incompressible torus by Claim 3.7 (and where the double branched cover of $\tau_{2}$ is Seifert fibered over the disk with two exceptional fibers).

Thus $M_{l}$ has a non-trivial torus decomposition, and [11] describes such manifolds with Heegaard genus 2. From now on, the labels (i)-(v) denote the five different types of such manifolds as described in [11, Theorem].

Claim 3.5 Assume $M$ is closed, connected, irreducible, has Heegaard genus 2, contains an essential torus, and is not a Seifert fiber space. If $M$ does not contain an essential non-separating torus, then the canonical torus decomposition of $M$ is one of types (i)-(iv). If $M$ contains an essential, non-separating torus, then the canonical torus decomposition of $M$ is of type (v), with the exception that one of the decomposing tori is removed if at least one of $M_{1}$ or $M_{2}$ is a product $T^{2} \times I$.

The canonical torus decomposition of $M$ has the property that any torus is isotopic into one of the pieces of the decomposition.

Proof This is the content of the proof of [11, Theorem]. When $M$ contains an essential, non-separating torus, [11] shows that $M$ has a decomposition of type (v). In this case, the identification described between the components of $\partial M_{1}$ and $\partial M_{2}$ guarantees that the decomposition is the canonical (minimal) torus decomposition, unless either $M_{1}$ or $M_{2}$ is $T^{2} \times I$. In that case, by amalgamating $M_{1}$ and $M_{2}$ we get a torus decomposition which must be minimal since $M$ is not a Seifert fiber space.

If $M$ does not contain a non-separating torus, then [11, proof of Theorem] shows that a canonical decomposition of $M$ is of one of the forms (i)-(iv).

A canonical torus decomposition (see for example [7]) has the property that any torus is isotopic into a piece of the decomposition (else there would be contiguous Seifert pieces where the fibers agree; contradicting the minimality of the decomposition).

We now argue that $M_{l}=N \cup_{T} S$ does not have a canonical torus decomposition of the form (i)-(v) guaranteed by Claim 3.5, thereby showing that $M_{l}$ cannot have Heegaard genus 2. Lemmas 4.2 and 4.4 of [11] show that the exterior of a two-bridge knot or link is atoroidal. Lemma 5.2 of [11] shows that the exterior of a one-bridge knot in a lens space of class $L_{K}$ of [11, Theorem] is atoroidal unless it is Seifert fibered over the Möbius band with a single exceptional fiber. In this case, the unique incompressible torus which is not boundary parallel is a vertical torus which bounds the neighborhood of a vertical Klein bottle. Finally, note that since $\left|j_{1}\right|, n>2, S$ is not the exterior of a two-bridge knot in $S^{3}$.

(i)-(ii) Assume there is a canonical decomposition of type (i) or (ii). $T$ is not isotopic to $\partial M_{1}$ since $N$ does not have tunnel number one by Claim 3.6 and since $S$ is not the 
exterior of a two-bridge knot. In a decomposition of type (i), since $M_{1}$ is atoroidal, $T$ must be an essential torus in $M_{2}$. By [11, Lemma 5.2] $M_{2}$ is Seifert fibered over a Möbius band with a single exceptional fiber, and $T$ bounds the neighborhood of a vertical Klein bottle in $M_{2}$. But this contradicts that $S$ is atoroidal and Seifert fibered over the disk (with a unique fibration). In a decomposition of type (ii), $M_{1}$ must be Seifert fibered over the Möbius band with at least one exceptional fiber, and $T$ must be vertical in this fibration. As $S$ is atoroidal, it must be the side of $T$ that lies in $M_{1}$. Then $N$ is the union of a circle bundle over a once-punctured Möbius band and $M_{2}$, where the circle fiber is identified with the meridian of $M_{2}$. This implies that $N$ has tunnel number one (one can find a tunnel for a two-bridge knot where two meridians represent jointly primitive curves in the genus- 2 handlebody). But this contradicts Claim 3.6.

(iii) Assume $M_{l}$ has a canonical decomposition of type (iii). If $T$ were isotopic to $\partial M_{1}$, then $M_{2}$ would have to be $N$ ( $S$ is not the exterior of a two-bridge knot in $S^{3}$ ). But $N$ is not tunnel number one. Thus $M_{1}$ must be Seifert fibered over the disk with three exceptional fibers and $T$ must be a vertical torus in $M_{1}$. Thus one side of $T$ is the union of a Seifert fiber space over the annulus with one exceptional fiber and $M_{2}$, where the Seifert fiber is identified with the meridian of the two-bridge knot exterior $M_{2}$. But such a manifold has tunnel number one (a meridian is primitive in the tunnel one handlebody of a two-bridge knot exterior). That is, both sides of $T$ have tunnel number one, contradicting Claim 3.6.

(iv) Assume that $M_{l}$ is decomposed as in (iv) into the three atoroidal manifolds $M_{1}, M_{2}, M_{3}$. Then $T$ is isotopic to a component of $\partial M_{3}$. But each side of $T$ has tunnel number one (eg $M_{3} \cup M_{2}$ has tunnel number one, since the Seifert fiber of $M_{2}$ is identified with the meridian of $M_{3}$, which is primitive in its tunnel one handlebody). This contradicts Claim 3.6.

(v) Assume $M_{l}$ is decomposed into $M_{1}$ and $M_{2}$ as in (v). The separating torus $T$ must be a vertical torus in $M_{1}$, where $M_{1}$ is Seifert fibered over an annulus with two exceptional fibers. Thus both sides of $T$ have tunnel number one, contradicting Claim 3.6 (note that the union of $M_{2}$ with the Seifert fiber space over the 3-punctured sphere has tunnel number one, since the Seifert fibers are identified with meridians of the two-bridge link, which are jointly primitive in its tunnel one handlebody).

This shows that $M_{l}$ does not have Heegaard genus 2, which contradicts our assumption that $M-\mathcal{N}\left(L_{1}\right)$ has tunnel number one, once we verify the supporting claims.

Claim 3.6 Let $\tau$ be the tangle pictured in Figure 8. The double branched cover $N$ of $\tau$ is irreducible, $\partial$-irreducible, and has tunnel number greater than one. 


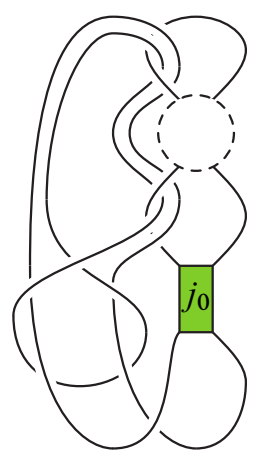

Figure 8

Proof Filling $\tau$ with the $\frac{1}{0}$-tangle gives the two-component unlink. Thus the corresponding Dehn filling of $N$ is $S^{2} \times S^{1}$.

The $\frac{0}{1}$-filling of $\tau$ gives the three component link $l^{\prime}$ in the first picture of Figure 9. That picture shows this link decomposed along a Conway sphere into two tangles $\tau_{1}, \tau_{2}$, where $\tau_{1}$ is the tangle of Figure 10 and where $\tau_{2}$ is the tangle gotten by taking a horizontal, unknotted circle along with two parallel, vertical arcs linking the circle. The double branched cover $M_{l^{\prime}}$ of $S^{3}$ along $l^{\prime}$ is the union along a torus $T^{\prime}$ of the double branched cover $N_{1}$ of $\tau_{1}$ and the double branched cover $N_{2}$ of $\tau_{2} . N_{2}$ is a twisted $I$-bundle over a Klein bottle which has exactly two descriptions as a Seifert fiber space corresponding to two different slopes on $\partial N_{2}$ : one as Seifert-fibered over a disk with two exceptional fibers of order 2, the other as a circle bundle over a Möbius band. By Claim 3.7, $N_{1}$ is irreducible, $\partial$-irreducible, and atoroidal. Thus $T^{\prime}$ is an incompressible torus in $M_{l^{\prime}}$, and either $M_{l^{\prime}}$ is a toroidal Seifert fiber space or $N_{1} \cup N_{2}$ is a non-trivial, canonical torus decomposition of $M_{l^{\prime}}$.

From the above discussion, the $\frac{1}{0}$-Dehn filling of $N$ is prime and the $\frac{0}{1}-$ Dehn filling of $N$ is irreducible, thus $N$ is irreducible. As the filling $M_{l^{\prime}}$ contains an essential torus, $N$ is not a solid torus. Thus $N$ is irreducible and $\partial$-irreducible.

Assume for contradiction that $N$ has tunnel number one. Then the toroidal $M_{l^{\prime}}$ has Heegaard genus 2.

First we show that $M_{l^{\prime}}$ is not a Seifert fiber space. Suppose it is. Then $N_{1}$ is a Seifert fiber space whose fiber is a fiber of one of the two Seifert fibrations of $N_{2}$, which is either the $\frac{1}{0}$-slope (as a Seifert fiber space over the disk) or the $\frac{0}{1}$-slope (as the Seifert fiber space over the Möbius band). However, the $\frac{0}{1}$-filling of $\tau_{1}$ is the unknot, implying that $N_{1}$ is the exterior of a knot in $S^{3}$ whose meridian is this $\frac{0}{1}$-slope. But the Seifert fiber of a knot exterior in $S^{3}$ is never meridional. Thus it must be that $N_{1}$ is a Seifert 

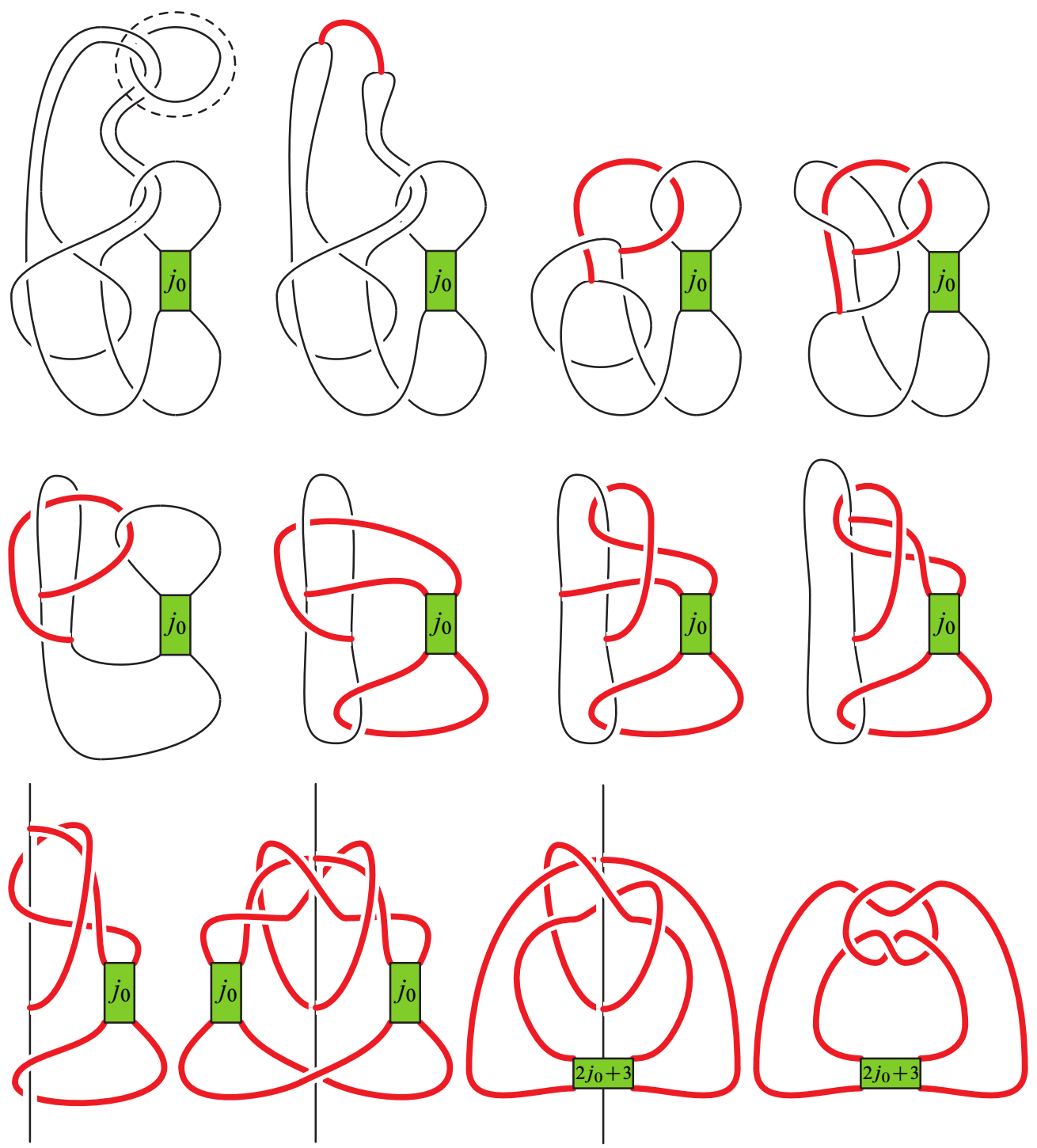

Figure 9

fibered knot exterior in $S^{3}$ whose fiber has slope $\frac{1}{0}$. Thus $M_{l^{\prime}}$ is a Seifert fibered space over the 2-sphere with four exceptional fibers, two of which have orders greater than 2 (by Claim 3.7, $N_{1}$ is not the exterior of a two-bridge knot). However, such a Seifert fibered space cannot have Heegaard genus 2. By [13], the splitting would have to be horizontal or vertical. It cannot be vertical because there are too many exceptional fibers. It cannot be horizontal by [16]. Thus $M_{l^{\prime}}$ is not a Seifert fiber space. 


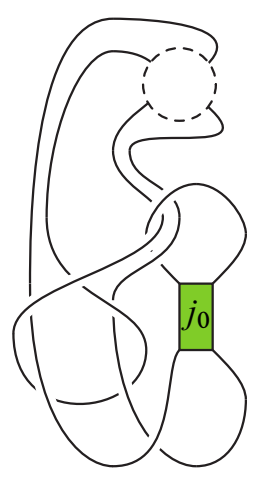

Figure 10

Thus $N_{1} \cup N_{2}$ is a non-trivial canonical decomposition of the genus- 2 manifold $M_{l^{\prime}}$. In particular, any torus in $M_{l^{\prime}}$ is isotopic to $T^{\prime}$. The main theorem of [11] describes the possible canonical decompositions of $M_{l^{\prime}}$ (see Claim 3.5). We rule out each of these possibilities. $\left(M_{1}, M_{2}, M_{3}\right.$ follow the notation of [11].)

(i) We rule out conclusion (i). By Claim 3.7, $N_{1}$ is not a Seifert fibered space unless $j_{0}=0$ (since $\left|j_{0}\right| \neq 1,2$ by hypothesis). In that case the slope of the regular fiber of $N_{1}$ on $T^{\prime}$ is $-\frac{1}{2}$, but this is not the slope of a lens space filling of $N_{2}$. Thus it must be that $M_{1}$ is $N_{2}$ and $M_{2}$ is $N_{1}$. That is, $N_{1}$ is the exterior of a one-bridge knot in a lens space whose meridian is identified along $T^{\prime}$ with the Seifert fiber, with slope $\frac{1}{0}$, of $N_{2}$ coming from its fibration over the disk. Filling $N_{1}$ along this meridian is a lens space. However, this filling is the double branched cover of the two-component link gotten by filling $\tau_{1}$ with the $\frac{1}{0}$-tangle. By Hodgson and Rubinstein [8], this link must be a two-bridge link. However, this is impossible since, for $j_{0} \neq 1,2$, one component of this link is knotted.

(ii) Conclusion (ii) does not hold since neither $N_{1}$ nor $N_{2}$ is a two-bridge knot exterior. (Claim 3.7 for $N_{1}$ and the fact that $N_{2}$ contains a Klein bottle.)

(iii) Conclusion (iii) does not hold as neither $N_{1}$ nor $N_{2}$ is a two-bridge knot exterior.

(iv) Conclusion (iv) does not hold as the torus decomposition of $M_{l^{\prime}}$ has only two pieces.

(v) Conclusion (v) does not hold as $M_{l^{\prime}}$ does not contain a non-separating torus.

This finishes the proof of Claim 3.6.

Claim 3.7 Let $\tau_{1}$ be the tangle in Figure 10. Let $N_{1}$ be the double cover of the tangle ball branched over $\tau_{1}$. Then $N_{1}$ is the exterior of the $\left(-2,3,2 j_{0}+3\right)$-pretzel knot 

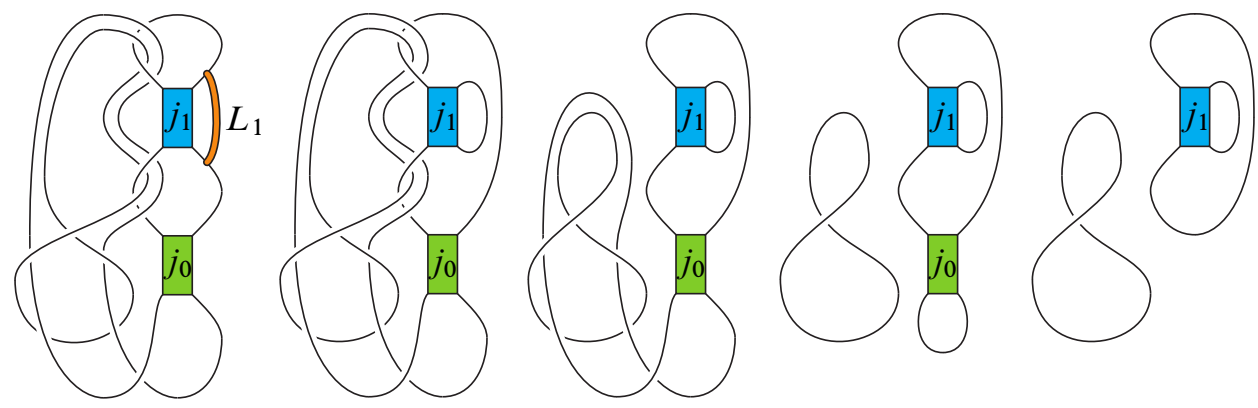

Figure 11

in $S^{3}$ pictured in the final picture of Figure 9. $N_{1}$ is irreducible, $\partial$-irreducible, and atoroidal for every $j_{0} . N_{1}$ is Seifert fibered only when $j_{0}=1,0,-1$, and when $j_{0}=0$ the Seifert fiber on $\partial N_{1}$ has slope $-\frac{1}{2} . N_{1}$ is the exterior of a two-bridge knot only when $j_{0}=-1,-2$.

Proof Figure 9 is a sequence of pictures identifying $N_{1}$ as the exterior of the nontrivial $\left(-2,3,2 j_{0}+3\right)$-pretzel knot. Since pretzel knots are not satellite knots [10], $N_{1}$ is irreducible, $\partial$-irreducible, and atoroidal for all $j_{0}$. These $\left(-2,3,2 j_{0}+3\right)$-pretzel knots are the $(3,5),(3,4)$, and $(2,5)$-torus knots when $j_{0}$ is $1,0,-1$, respectively, and hyperbolic otherwise [10]. Moreover, they are two-bridge knots only when $j_{0}=-1,-2$; see eg [9]. When $j_{0}=0$, the slope of the Seifert fiber on $\partial N_{1}$ may be determined as the slope of the tangle that fills $\tau_{1}$ of Figure 10 to produce the connected sum of the $(3,1)$ and $(-4,1)$ torus knots.

(4) Filling $M-\mathcal{N}\left(L_{1}\right)$ along the slope $\partial \hat{\boldsymbol{R}}$ is atoroidal The $R$-framing of $L_{1} \subset S^{3}$ is the $\hat{R}$-framing of $L_{1} \subset M$ and corresponds to the page framing of the arc $L_{1}$ at the end of Figure 6 . Therefore the $\hat{R}$-framed surgery on $L_{1} \subset M$ is the double branched cover of the link in the second picture of Figure 11. The subsequent pictures show isotopies of this link to the split link comprised of the unknot and the $\left(2, j_{1}\right)$-torus link. Thus the $\hat{R}$-framed surgery on $L_{1} \subset M$ is homeomorphic to $S^{1} \times S^{2} \# L\left(j_{1}, 1\right)$, which is atoroidal.

(3) $L_{1}$ is not the cable of a tunnel number one knot in $M$ for which the cabling annulus has the same slope on $L_{1}$ as $\partial \hat{\boldsymbol{R}} \quad$ Suppose $L_{1}$ is cabled as described about the tunnel number one knot $J$ in $M$. Let $A_{1}$ be the cabling annulus, properly embedded in $M-\mathcal{N}\left(L_{1}\right)$, whose two boundary components have the same slope on $L_{1}$ as $\partial \widehat{R}$ (the 0 -slope).

First assume $\left|j_{1}\right|=1$. As $L_{1}$ is cabled, the 0 -surgery in $M$ along $L_{1}$ will produce a manifold with a lens space (of positive, finite order in first homology) summand. But we saw above that 0 -surgery on $L_{1}$ produces $S^{1} \times S^{2} \# L\left(\left|j_{1}\right|, 1\right)$, which is $S^{1} \times S^{2}$. 
Thus we may assume that $\left|j_{1}\right|>1$. As $\left|j_{0}\right|,\left|j_{1}\right| \geq v, M$ is hyperbolic. Because $L_{1}$ is isotopic to a meridian of $J_{1}$ (see Figure 5), $L_{1}$ is also a $\left(j_{1}, 1\right)$-cable of $J_{1}^{\prime}$, the dual to the $-1 / j_{1}-$ surgery on $J_{1}$. Furthermore, the slope of this cabling annulus $A_{2}$ on $L_{1}$ is that of $\partial \hat{R}$. Now the exterior of $J_{1}^{\prime}$ in $M$ is $N$, the double branched cover of $\tau$ in Claim 3.6. By that claim, $J_{1}^{\prime}$ does not have tunnel number one. Thus $J$ is not isotopic to $J_{1}^{\prime}$ in $M$.

We may isotope $A_{1}, A_{2}$ in $M-\mathcal{N}\left(L_{1}\right)$ so that they intersect in parallel essential curves in the interiors of $A_{1}$ and $A_{2}$. Let $V_{i}$ be the cabling solid torus for $A_{i}$ in the exterior of $L_{1}$. Note that any incompressible annulus in $V_{1}$ is $\partial$-parallel. Hence for $\{i, j\}=\{1,2\}$, we may assume that $\partial A_{i}$ lies outside of $V_{j}$, and that each component of $A_{i} \cap V_{j}$ is parallel to $\partial \mathcal{N}\left(L_{1}\right) \cap V_{j}$. First assume that $A_{1} \cap A_{2}$ is non-empty. Then $V_{j}-\mathcal{N}\left(A_{i}\right)$ consists of solid tori, exactly one of which, $\mathcal{C}_{j}$, has the property that $A_{i} \cap \mathcal{C}_{j}$ is not longitudinal. The core of $\mathcal{C}_{j}$ is isotopic to the core of $V_{j}$. If $\mathcal{C}_{j}$ lies in $V_{i}$ then $\mathcal{C}_{j}=\mathcal{C}_{i}$ and $J$ would be isotopic to $J_{1}^{\prime}$. So it must be that $\mathcal{C}_{j}$ lies outside of $V_{i}$. As $\mathcal{C}_{j}$ meets $V_{i}$ in a subannulus of $A_{i}, \mathcal{T}=\mathcal{C}_{j} \cup V_{i}$ is a Seifert fiber space over the disk with two exceptional fibers. Note that $L_{1}$ is isotopic to a regular fiber of $\mathcal{T}$. If the boundary of $\mathcal{T}$ is compressible in $M$, then either it, and hence $L_{1}$, is contained in a ball, or $M$ is either a small Seifert fiber space or the connected sum of lens spaces. If its boundary is incompressible, then $M$ is toroidal. As $M$ is hyperbolic, it must be that $L_{1}$ lies in a ball in $M$. Then $M$ is a connected summand of the 0 -surgery on $L_{1}$. But $M$ is hyperbolic, and 0 -surgery on $L_{1}$ produces $S^{2} \times S^{1} \# L\left(\left|j_{1}\right|, 1\right)$.

Thus it must be that $A_{1}, A_{2}$ are disjoint. Then $V_{1} \cup \mathcal{N}\left(L_{1}\right) \cup V_{2}$ is a Seifert fiber space over the disk with two exceptional fibers, of which $L_{1}$ is a regular fiber. As above, this contradicts the hyperbolicity of $M$.

Teragaito also describes the link $\mathcal{L}_{1,1}^{\prime}$ in [17] and states that 0 -surgery $(+4$-surgery with respect to the Seifert framing) on each of the knots $K_{1,1}^{\prime n}$ yields the same Seifert fibered manifold of type $S^{2}(3,4,8)$. We observe this as follows: Continuing from Figure 6, Figure 12 shows that setting $j_{0}=j_{1}=1$ produces a link isotopic to the Montesinos link $m\left(0 ;-\frac{1}{3}, \frac{5}{8},-\frac{1}{4}\right)$. The double branched cover of this link is the manifold $M_{1,1}$ that results from the 0 -surgery on $K_{1,1}^{\prime 0}$ and is a Seifert fibered manifold of the type claimed.

Theorem 3.8 For the second Teragaito family, where $j_{0}=1$ and $j_{1}=1,\left\{\mathfrak{b}_{2}\left(K^{n}\right)\right\}$ is finite.

Proof We set $j_{0}=1$ and $j_{1}=1$. Figure 12 also keeps track of the orange arc that lifts to $L_{1}$. The final link of this figure is decomposed in Figure 13 into two 3 -strand trivial 

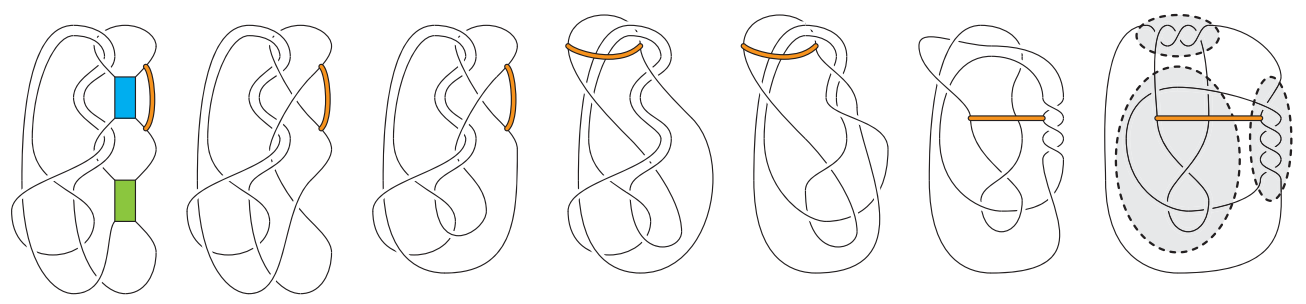

Figure 12
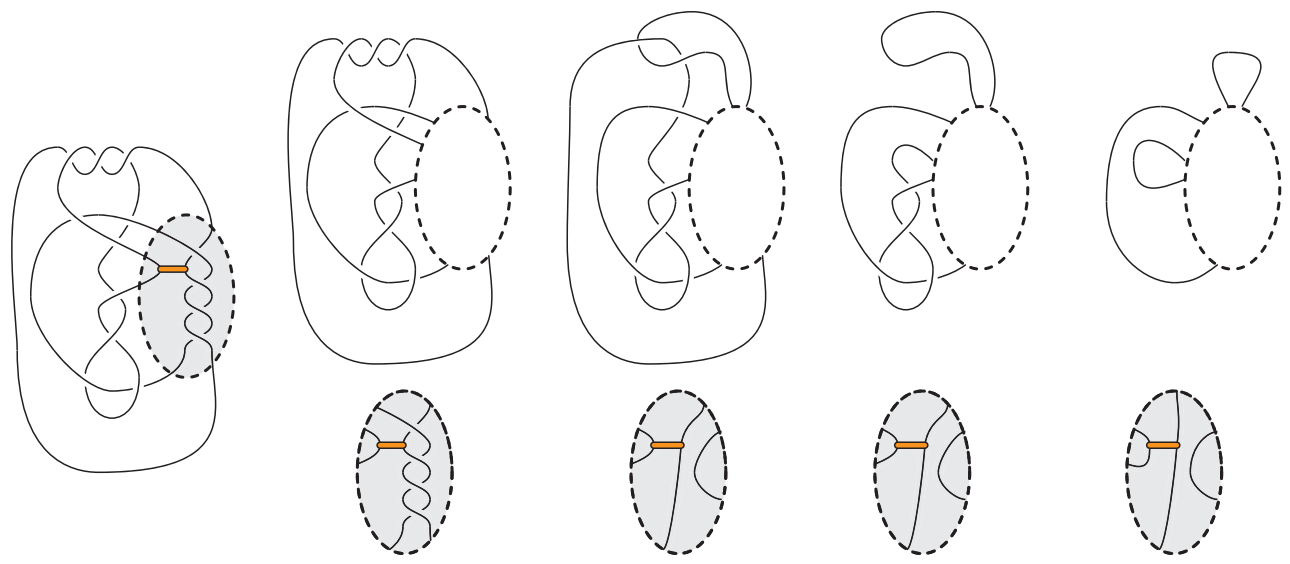

Figure 13

tangles, one of which contains the orange arc as a "core arc". That is, in the genus- 2 handlebody that is the double branched cover of this 3-strand trivial tangle, the orange arc lifts to a core. Consequently, this implies $L_{1}$ is a core curve of a genus- 2 splitting of $M$. Hence the tunnel number of $L_{1} \subset M$ is one. Moreover, if $\hat{F}$ is a Heegaard surface of this splitting, then $L_{1}$ may be isotoped into $\hat{F}$ with any desired framing. Therefore there is an isotopy of the annulus $\hat{R}$ into $\hat{F}$. As argued at the end of the proof of Corollary 1.1, there is an upper bound for $\mathfrak{b}_{\widehat{F}}\left(K^{n}\right)$ and hence for $\mathfrak{b}_{2}\left(K^{n}\right)$ as well.

Definition 3.9 A non-orientable, closed surface with Euler characteristic -1 is called a Dyck's surface.

Lemma 3.10 For infinitely many pairs $\left(j_{0}, j_{1}\right)$ the manifold $M_{j_{0}, j_{1}}$ is hyperbolic and contains no Dyck's surface.

Proof Recall that $M_{j_{0}, j_{1}}$ is obtained by Dehn surgery on the link $K^{\prime} \cup J_{0} \cup J_{1}$ in $S^{3}: 0,-1 / j_{0},-1 / j_{1}-$ surgery on $K^{\prime}, J_{0}, J_{1}$ respectively. Let $X$ be the exterior 
of $K^{\prime} \cup J_{0} \cup J_{1}$ in $S^{3}$ and $T, T_{0}, T_{1}$ be the components of $\partial X$ corresponding to $K^{\prime}, J_{0}, J_{1}$, respectively. Recall that $W_{0}$ is the 0 -filling of $X$ along $T$ and that the interiors of both $X$ and $W_{0}$ are hyperbolic of finite volume.

First we note that $M_{0,1}$ contains no Dyck's surface, Klein bottle, or projective plane [1, appendix] $\left(M_{0,1}\right.$ is the Seifert fiber space $\left.S^{2}\left(-\frac{1}{2}, \frac{1}{6}, \frac{2}{7}\right)\right)$. As $M_{0,1}$ is a filling of $W_{0}$, this implies that $W_{0}$ contains no Dyck's surface.

Claim 3.11 For $\left|j_{1}\right|>108$, the manifold $W_{0}\left(j_{1}\right)$ obtained by $-1 / j_{1}-$ filling the $T_{1}$ boundary of $W_{0}$ contains no Dyck's surface.

Proof Assume $\left|j_{1}\right|>108$. The obvious disk that $K^{\prime}$ bounds in the final picture of Figure 5 gives rise in $W_{0}$ to a 4 -punctured disk with punctures on $T_{1}$. By tubing an appropriate pair of these punctures we get a 2-punctured torus $Q^{\prime}$ properly embedded in $W_{0}$ whose two boundary components are coherently oriented curves representing meridians (slope $\frac{1}{0}$ ) of $J_{1}$. Assume for contradiction there is a Dyck's surface $S$ in $W_{0}\left(j_{1}\right)$. Let $J_{1}^{\prime}$ be the core of the attached solid torus at $T_{1}$ in $W_{0}\left(j_{1}\right)$. Isotope $S$ in $W_{0}\left(j_{1}\right)$ to intersect $J_{1}^{\prime}$ minimally. Let $S^{\prime}=W_{0} \cap S$. As $W_{0}$ contains no Dyck's surface, $\partial S^{\prime}$ is a non-empty collection of curves of slope $-1 / j_{0}$ on $T_{1}$. Isotope $\partial Q^{\prime}, \partial S^{\prime}$ to intersect minimally in $W_{0}$. Then no arc of $Q^{\prime} \cap S^{\prime}$ is boundary parallel in either $Q^{\prime}$ or $S^{\prime}$ (note that a boundary parallel arc in $Q^{\prime}$ is orientation-preserving so the parity rule still applies). Let $A$ be the punctured genus- 2 surface coming from a regular neighborhood of $S^{\prime}$ in $W_{0}\left(j_{1}\right)$. Then no arc of $Q^{\prime} \cap A$ is boundary parallel in $Q^{\prime}$ or in $A$. Consider the graphs of intersection $G_{A}, G_{Q^{\prime}}$ coming from the arcs of $Q^{\prime} \cap A$. Then $G_{A}, G_{Q^{\prime}}$ have no monogons.

As the distance between the slopes of the boundaries of $Q^{\prime}$ and $A$ on $T_{1}$ is $\left|j_{1}\right|, G_{Q^{\prime}}$ has $\left|j_{1}\right||\partial A|$ edges. Let $\widetilde{G_{Q^{\prime}}}$ be the reduced graph of $G_{Q^{\prime}}$ gotten by amalgamating parallel edges of $G_{Q^{\prime}}$. The proof of Claim 2.9 shows that $\widetilde{G_{Q^{\prime}}}$ has at most six edges. Thus $G_{Q^{\prime}}$ must have a collection $\mathcal{E}$ of at least $\left|j_{1}\right||\partial A| / 6$ parallel edges. Let $G_{A}(\mathcal{E})$ be the subgraph of $G_{A}$ corresponding to these edges (along with all vertices of $G_{A}$ ). Then the valence of each vertex of $G_{A}$ is at least $\left|j_{1}\right| / 6>18$. By Claim 2.10, two of the edges of $G_{A}(\mathcal{E})$ are parallel on $G_{A}$. As in the proof of Lemma 2.8, the union of the disks bounded in $Q^{\prime}, A$ by an innermost pair of edges gives rise to a Möbius band properly embedded in $W_{0}$. But this contradicts the hyperbolicity of $W_{0}$.

Claim 3.12 Assume $W_{0}\left(j_{1}\right)$ is hyperbolic. If $\left|j_{1}\right|>108$ and $M_{j_{0}, j_{1}}$ and $M_{j_{0}^{\prime}, j_{1}}$ both contain Dyck's surfaces, then $\left|j_{0}-j_{0}^{\prime}\right| \leq 324$.

Proof Assume for contradiction that $\left|j_{1}\right|>108,\left|j_{0}-j_{0}^{\prime}\right|>324$ and that $S \subset M_{j_{0}, j_{1}}$ and $F \subset M_{j_{0}^{\prime}, j_{1}}$ are embedded Dyck's surfaces. Isotope $S, F$ so that they intersect 
the core of the solid torus attached to $T_{0}$ in $M_{j_{0}, j_{1}}, M_{j_{0}^{\prime}, j_{1}}$ minimally. Let

$$
S^{\prime}=S \cap W_{0}\left(j_{1}\right), \quad F^{\prime}=F \cap W_{0}\left(j_{1}\right) .
$$

By Claim 3.11, $\partial S^{\prime}$ is a non-empty collection of curves of slope $-1 / j_{0}$ and $\partial F^{\prime}$ is a non-empty collection of curves of slope $-1 / j_{1}$ on $T_{2}$ in $\partial W_{0}\left(j_{1}\right)$. Isotope $S^{\prime}, F^{\prime}$ to intersect minimally. Then no arc of $S^{\prime} \cap F^{\prime}$ is boundary parallel in either $S^{\prime}$ or $F^{\prime}$. Let $A$ and $B$ be the boundaries of regular neighborhoods of $S^{\prime}$ and $F^{\prime}$ in $W_{0}\left(j_{1}\right)$, respectively. Then $A$ and $B$ are both punctured surfaces of genus 2. Consider the graphs of intersection $G_{A}, G_{B}$. Neither $G_{A}$ nor $G_{B}$ have monogons. The valence of each vertex of $G_{A}$ is $\left|j_{0}-j_{0}^{\prime}\right||\partial B|$. Let $\widetilde{G_{A}}$ be the reduced graph of $G_{A}$. By Claim 2.10, the valence of some vertex of $\widetilde{G_{A}}$ is at most 18 . This implies that there must be a group of parallel edges $\mathcal{E}$ in $G_{A}$ with cardinality $\left|j_{0}-j_{0}^{\prime}\right||\partial B| / 18$. Let $G_{B}(\mathcal{E})$ be the subgraph of $G_{B}$ gotten from the edges corresponding to $\mathcal{E}$. The vertices of $G_{B}(\mathcal{E})$ have valence $\left|j_{0}-j_{0}^{\prime}\right| / 18>18$. Thus again Claim 2.10 implies that two edges of $G_{B}(\mathcal{E})$ are parallel in $G_{B}$. Once again, the disks bounded on $A, B$ by an innermost pair of such edges gives rise to a Möbius band properly embedded in $W_{0}\left(j_{1}\right)$. But this contradicts that $W_{0}\left(j_{1}\right)$ is hyperbolic.

Recall that $M_{j_{0}, j_{1}}$ is hyperbolic as long as $\left|j_{0}\right|,\left|j_{1}\right| \geq v$. As $W_{0}$ is hyperbolic, there is a constant $v^{\prime}$ such that if $\left|j_{1}\right|>v^{\prime}$ then $W_{0}\left(j_{1}\right)$ is hyperbolic. Fix $\left|j_{1}\right|>\max \left\{v, v^{\prime}, 108\right\}$. By Claim 3.11, for all but finitely values of $j_{0}, M_{j_{0}, j_{1}}$ will be hyperbolic and contain no Dyck's surface. This completes the proof of Lemma 3.10.

\section{References}

[1] K L Baker, C Gordon, J Luecke, Obtaining genus-2 Heegaard splittings from Dehn surgery, Algebr. Geom. Topol. 13 (2013) 2471-2634 MR3116298

[2] K L Baker, C Gordon, J Luecke, Bridge number, Heegaard genus and non-integral Dehn surgery, Trans. Amer. Math. Soc. 367 (2015) 5753-5830 MR3347189

[3] M Culler, N M Dunfield, J R Weeks, SnapPy, a computer program for studying the geometry and topology of 3-manifolds Available at http://snappy.computop.org

[4] M Culler, C M Gordon, J Luecke, P B Shalen, Dehn surgery on knots, Bull. Amer. Math. Soc. 13 (1985) 43-45 MR788388

[5] D Gabai, Foliations and the topology of 3-manifolds, III, J. Differential Geom. 26 (1987) 479-536 MR910018

[6] C M Gordon, Boundary slopes of punctured tori in 3-manifolds, Trans. Amer. Math. Soc. 350 (1998) 1713-1790 MR1390037 
[7] A Hatcher, Notes on basic 3-manifold topology (2007) lecture notes Available at http: //www. math. cornell. edu/ hatcher/3M/3Mfds.pdf

[8] C Hodgson, J H Rubinstein, Involutions and isotopies of lens spaces, from: "Knot theory and manifolds", (D Rolfsen, editor), Lecture Notes in Math. 1144, Springer, Berlin (1985) 60-96 MR823282

[9] A Kawauchi, A survey of knot theory, Birkhäuser, Basel (1996) MR1417494

[10] D Kim, J Lee, Some invariants of pretzel links, Bull. Austral. Math. Soc. 75 (2007) 253-271 MR2312569

[11] T Kobayashi, Structures of the Haken manifolds with Heegaard splittings of genus two, Osaka J. Math. 21 (1984) 437-455 MR752472

[12] T Mattman, K Miyazaki, K Motegi, Seifert-fibered surgeries which do not arise from primitive/Seifert-fibered constructions, Trans. Amer. Math. Soc. 358 (2006) 4045-4055 MR2219009

[13] Y Moriah, J Schultens, Irreducible Heegaard splittings of Seifert fibered spaces are either vertical or horizontal, Topology 37 (1998) 1089-1112 MR1650355

[14] J K Osoinach, Jr, Manifolds obtained by surgery on an infinite number of knots in $S^{3}$, Topology 45 (2006) 725-733 MR2236375

[15] M Scharlemann, Sutured manifolds and generalized Thurston norms, J. Differential Geom. 29 (1989) 557-614 MR992331

[16] E Sedgwick, The irreducibility of Heegaard splittings of Seifert fibered spaces, Pacific J. Math. 190 (1999) 173-199 MR1722770

[17] M Teragaito, A Seifert fibered manifold with infinitely many knot-surgery descriptions, Int. Math. Res. Not. 2007 (2007) MR2347296

Department of Mathematics, University of Miami

1365 Memorial Drive, Coral Gables, FL 33146, USA

Department of Mathematics, University of Texas at Austin

2515 Speedway Stop C1200, Austin, TX 78712-1202, USA

Department of Mathematics, University of Texas at Austin

2515 Speedway Stop C1200, Austin, TX 78712-0257, USA

k.baker@math.miami.edu, gordon@math.utexas.edu,

luecke@math.utexas.edu

Received: 20 December 2013 Revised: 11 February 2015 